\title{
Il motivo dell'aspidochelone nella tradizione letteraria del Physiologus. Considerazioni esegetiche e storico-religiose
}

\author{
Fausto IANNELLO \\ Università degli Studi di Messina \\ faustoiannello@gmail.com
}

RIASSUNTO: Scopo di questo contributo è quello di ricostruire e comparare il motivo dell'enigmatico animale chiamato aspidochelone isolatamente nel Physiologus greco e in tutte le successive versioni antiche e medievali. Questo approccio di analisi può essere utile a porre più nettamente in risalto le significazioni religiose che in principio l'oscuro autore alessandrino volle velare dietro l'ibrida creatura. Si procederà, pertanto, con un'analisi storico-letteraria e, successivamente, esegetica dei riadattamenti e delle manifestazioni dell'aspidochelone nelle diverse versioni per rilevarne continuità, analogie o differenze, di carattere sia formale sia sostanziale.

\section{The Motif of the aspidochelone in the Literary Tradition of the Physiologus. Exegetical and Historical-Religious Aspects}

\begin{abstract}
The aim of this article is to reconstruct the motif of the enigmatic animal called aspidochelone first in the Greek Physiologus and then to compare its use in all the ancient and medieval versions that followed. This comparative approach may prove useful as a way to bring more clearly to light the religious meanings that the unknown Alexandrian author wanted to conceal from the outset behind the hybrid creature. Therefore, we will carry out a historico-literary and then exegetical analysis of the succesive adaptations and outlooks showed by the aspidochelone in the different versions, so as to point out both formal and substantial continuities, analogies or differences.
\end{abstract}

Parole-Chiave: Physiologus, aspidochelone, bestiario, simbolismo degli animali, Scuola Alessandrina, esegesi cristiana.

KEYWORDs: Physiologus, aspidochelone, bestiary, animal symbolism, Alexandrian School, Christian exegesis.

FECHA DE RECEPCIÓN: 7 de julio de 2011.

FECHA DE ACEPTACIÓN: 12 de noviembre de 2011. 



\title{
Il motivo dell'aspidochelone nella tradizione letteraria del Physiologus. Considerazioni esegetiche e storico-religiose
}

\author{
Fausto IANNELLO
}

\section{Premessa e note introduttive}

Noto e fortunato come pochi altri tra quelli del Physiologus, il motivo dell'aspidochelone — creatura fantastica nella quale, come vedremo, si opera l'incontro di alcune precise nature e simbologie animali- nei secoli è stato oggetto di traduzioni più o meno libere, varianti, riadattamenti e riletture in ambiti e generi storico-letterari e storico-religiosi lontani nel tempo, nello spazio, nonché su un piano meramente culturale, che nondimeno non sembrano avere inficiato la sopravvivenza delle funzioni allegoriche originarie e di quegli elementi di esegesi cristiana che in principio l'autore alessandrino volle esprimere nell'ibrida bestia. È pertanto questa continuità funzionale che in primo luogo si intende dimostrare nel presente contributo, ricorrendo a un approccio comparativo che, come fin qui non è mai stato compiuto in uno specifico studio sul motivo in questione, raccolga e ponga a confronto gli episodi dell'aspidochelone nelle diverse redazioni e versioni del Physiologus (greche, orientali, latine, romanze, germaniche) $)^{1}$ e le sue utilizzazioni in quei testi che ne tradiscano un'influenza diretta dall' età tardoantica a quella medievale e umanistica confluita soprattutto nei bestiari.

Non pervenutoci nella redazione originaria, il Physiologus greco fu con buona probabilità messo per iscritto tra la fine del II e i primi decen-

\footnotetext{
${ }^{1}$ Non saranno considerate le versioni slave e romene (strettamente connesse nella tradizione manoscritta) per due semplice ragioni: i codici pervenutici sono perlopiù molto tardi (XVI sec. per le versioni serbe e addirittura il XVIII per quelle romene) e l'aspidochelone o non è compreso nelle singole versioni (russa, serba I, romena) o la traduzione segue quasi alla lettera le redazioni greche (ceca, serba II). Si tenga presente, inoltre, che altra peculiarità delle versioni slave è quella di aggiungere diversi animali estranei alla tradizione fisiologica. Si veda lo studio specifico di Mirandola, 2001; cf. pure Dolcetti Corazza, 1992, pp. 39-48.
} 
ni del III secolo ad Alessandria. ${ }^{2}$ Nulla di certo può dirsi sul suo autore, nei manoscritti più antichi semplicemente definito Physiologus. ${ }^{3}$ Come terminus ante quem si potrebbe stabilire il 254, l'anno in cui si spense Origene, che, sulla scorta di Filone e Clemente, già mostrava di conoscere una tradizione fisiologica piuttosto definita. ${ }^{4}$ Questa datazione può effettivamente essere più alta. Friedrich Lauchert, infatti, ha proposto di situare la composizione originaria negli anni intorno al 165, data di morte di Giustino, che da alcuni indizi sembrerebbe manifestarne una qualche conoscenza; ma in uno studio più recente e specifico, Alan Scott mostra di preferire ancora la seconda metà del III secolo, ovvero gli anni appena successivi alla morte di Origene. ${ }^{5}$

Il primo a menzionare espressamente l'opera fu Rufino nel 407/408, per quanto si tratti di un riferimento alquanto sfuggente: Nam physiologus de catulo leonis haec scribit, quod cum natus fuerit, tribus diebus ac tribus noctibus dormiat; tum deinde patris fremitu uel mugitu tamquam tremefactus cubilis locus suscitet catulum dormientem. ${ }^{6}$ Sarà poi citata nel 494 nel Decretum Gelasianum (de libris recipiendis et non recipiendis) - anche se quasi sicuramente questo fu redatto nella Gallia meridionale tra la fine del v sec. e i primi anni del VI- nella sezione quinta, dal titolo Cetera quae ab hereticis sive scismaticis conscripta vel praedicata sunt, nullatenus recipit catholica et apostolica Romana ecclesia; e quibus pauca, quae ad memoriam venerunt et a catholicis vitanda sunt [...], leggiamo Liber Physiologus ab hereticis conscriptus et beati Ambrosii nomine praesignatus, apocryphus. ${ }^{7}$ Nonostante ciò, e di là da qualche spunto, il documento non può più essere inserito nel novero delle opere ereticali, come hanno chiarito gli esperti ${ }^{8}$ in ogget-

${ }^{2}$ Vedi Zambon, 2002, p. 15; alla fine del IV secolo, in un contesto esegetico di matrice origeniana, lo assegna invece Maria Pia Ciccarese (in Ciccarese, 2002, p. 13).

${ }^{3}$ Cf. Lauchert, 1974, pp. 3-4.

${ }^{4}$ Vedi la discussione in Zambon, 2002, pp. 15 ss.

${ }^{5}$ Scott, 1998.

${ }^{6}$ De benedictionibus patriarcharum, I 6,1.20 (ed. M. Simonetti, CChrSL, 20).

${ }^{7}$ Cap. V, Dobschütz, 1912. Il testo di Dobschütz è riportato anche in PL 59, 157-180, dove si pubblicano solo gli ultimi due capitoli del documento, ossia il quarto e il quinto, poiché considerati indipendenti e successivi rispetto ai primi tre, risalenti probabilmente al pontificato di Damaso (366-384); si veda Grossi, 2001, pp. 231-255.

${ }^{8}$ Cf. Zambon, 2002,pp. 16-17. 
tiva conformità a un passaggio dello stesso Physiologus, nel quale, a proposito dell'abitudine dell'airone di vivere sempre negli stessi luoghi (47, 7-11), così stabilisce:

Anche tu, o fedele, non cercare i molti luoghi degli eretici: tuo solo nido sia la santa Chiesa di Dio e tuo solo nutrimento il pane disceso dal cielo, il Signore nostro Gesù Cristo, e non toccare i morti insegnamenti, se vuoi ricevere il ben cotto pane celeste, e non cercare i molti luoghi degli eretici. ${ }^{9}$

Di certo l'autore ricorre al termine physiologia sulla scorta di quell'allegoria alessandrina che, con Filone, assegnava alla "conoscenza della natura delle cose" un ruolo privilegiato nel processo di indagine delle "cose celesti", di quelli eventi che si verificano e nell'aria e nel cielo. La "fisiologia" diviene allegoria, ${ }^{10}$ intelligenza spirituale degli insegnamenti scritturistici, e colui che è iniziato allo studio della natura ( $\varphi v \sigma ı \lambda o \gamma i ́ \alpha)$ può giungere alla scienza, ${ }^{11}$ poiché

per mezzo della sapienza ci è assicurata la più eccellente delle migrazioni, consistente nell'uscita dell'anima dalla astronomia per fare ingresso nella vera scienza della natura [ $\left.\varphi v \sigma i \lambda_{0} \gamma^{\prime} \alpha\right]$; nell'uscita dalla insicura congettura verso una comprensione chiara, e, per dirla giusta, fuori dal creato verso l'increato, fuori dal mondo verso il Creatore e Padre di esso. ${ }^{12}$

In sostanza, Filone vuole rendere chiaro che il fine ultimo della

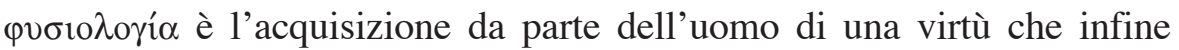
lo faccia pervenire alla conoscenza del Creatore in un atto di offerta e consacrazione di se stesso. ${ }^{13}$ Analogamente, Clemente considera la fisiologia come conoscenza che innalza l'uomo alla contemplazione del divino, mezzo per ricevere l'iniziazione ai piccoli e ai grandi misteri: la

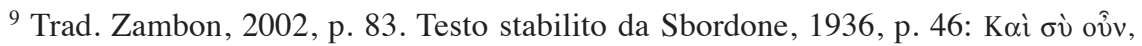

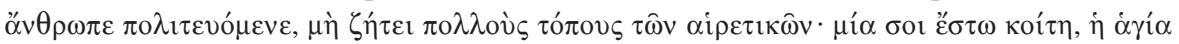

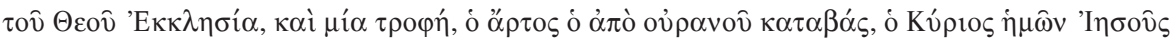

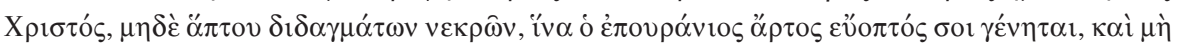

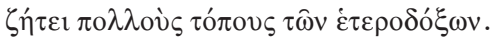

${ }^{10}$ De somniis, I, 120.

${ }^{11}$ De Cherubim, 121 (cfr. 4 e 87).

${ }^{12}$ Qui rerum divinarum heres sit, 98 (trad. di R. Radice); vedi anche De ebrietate, 91-92; De somniis, I 184; De aeternitate mundi, 138.

${ }^{13}$ De mutatione nominum, 73; 76; 220. 
"scienza della natura", conducendo l'uomo dall'osservazione delle realtà cosmogoniche alle verità teologiche, diventa allora acquisizione delle verità celesti attraverso la contemplazione e lo studio del Creato.$^{14}$ È una concezione di fatto già presente in san Paolo, che così condensa quella visione platonico-cristiana che vede nel mondo sensibile l'ombra o il riflesso di realtà soprasensibili e divine: ${ }^{15}$

Invisibilia enim ipsius, a creatura mundi, per ea quae facta sunt, intellecta, conspiciuntur: sempiterna quoque eius virtus, et divinitas. ${ }^{16}$

Videmus nunc per speculum in aenigmate: tunc autem facie ad faciem. Nunc cognosco ex parte: tunc autem cognoscam sicut et cognitus sum. ${ }^{17}$

E nell'oscuro personaggio del Physiologus, come notano Max Wellmann ${ }^{18}$ e Francesco Zambon, ${ }^{19}$ si può arrivare a riconoscere un ispirato rivelatore di conoscenze tradizionali intorno ai segreti della natura, un

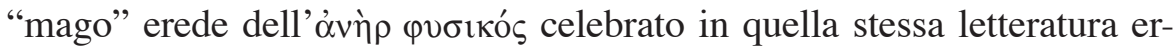
metica greco-egizia che, combinata con le nuove dottrine ebraiche e con le speculazioni cristiche, ha generato il Physiologus. Fuor di dubbio è ormai anche l'influenza delle speculazioni sul simbolismo degli animali, delle pietre e delle piante ${ }^{20}$ contenute nei manuali di geroglifici di autori quali Manetone (323-254 a.C.), Bolo di Mendes (III-II sec. a. C.) e Cheremone (I sec. d. C.), pervenutici in età tarda (sec. v) nella nota opera di Orapollo, Hieroglyphica. ${ }^{21}$ Ma l'autore che si nasconde dietro il Physiologus è soprattutto un "esegeta della natura secondo i canoni della fede cristiana", ${ }^{22}$ non una fonte del simbolismo animale; il suo è un manuale di simboli che, sulla scorta dell'esegesi tripartita origeniana (letterale,

${ }^{14}$ Stromata, IV 1.

${ }^{15}$ Cf. Zambon, 2003, p. 23.

${ }^{16}$ Rom., 1, 20.

171 Cor., 13, 12.

${ }^{18}$ Wellmann, 1930, pp. 3-4.

${ }^{19}$ Zambon, 2002, pp. 24-25.

${ }^{20}$ A prova di ciò, si tenga presente che nella redazione greca sono comprese 48 "nature": 41 animali, 5 pietre, 2 alberi.

${ }^{21}$ Il primo studioso a parlare concretamente e con competenza di influenze egizie fu Wellmann, 1930; vedi anche Iversen, 1961, p. 48, e Zambon, 2002, pp. 23 ss.

${ }^{22}$ Sbordone, 1936, p. 174 
morale, spirituale) ${ }^{23}$ fornisce in successione una descrizione della natura dell'animale e un'interpretazione etica e teologica. ${ }^{24}$ Scrive Maria Pia Ciccarese che "non v'è alcun dubbio sulla natura esegetica dell'opera. Il punto di partenza è sempre un testo biblico, prima citato alla lettera e poi interpretato secondo le norme dell'allegoria. Sia il metodo ermeneutico che i risultati dell'interpretazione non divergono da quelli che conosciamo [...] da Clemente ad Agostino". ${ }^{25}$ Così intesa, la fisiologia, per mezzo dell'acquisizione delle nature dei singoli animali, diviene iniziazione alla completa intelligenza delle Scritture. ${ }^{26}$

\section{La redazione greca}

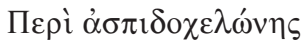

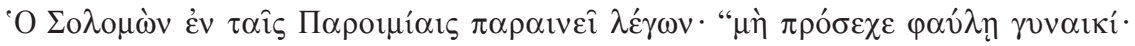

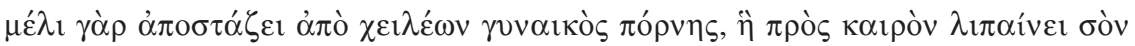

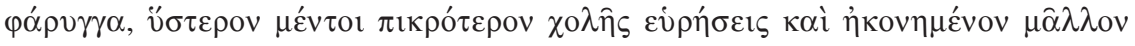

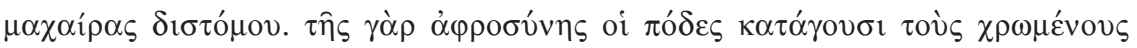

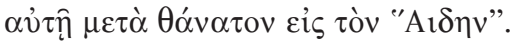

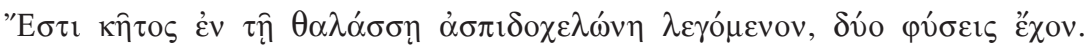

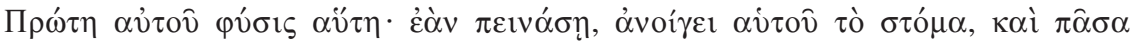

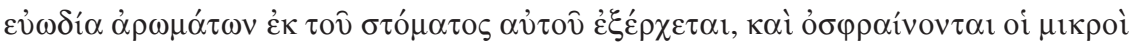

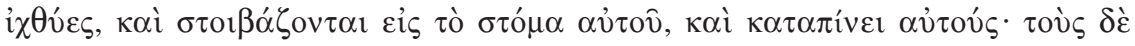

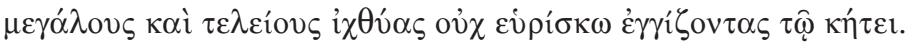

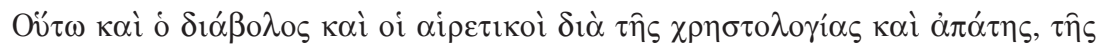

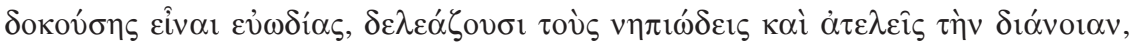

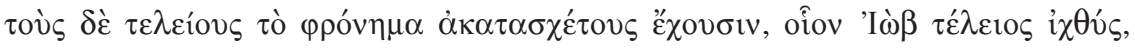

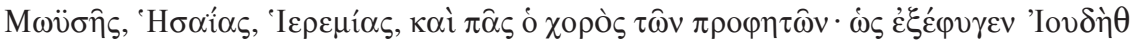

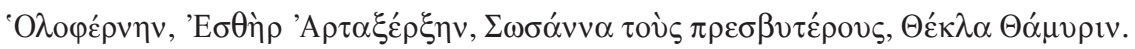

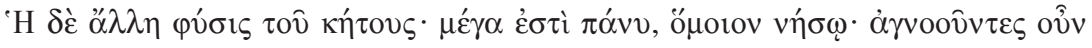

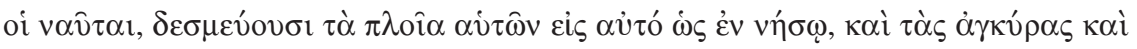

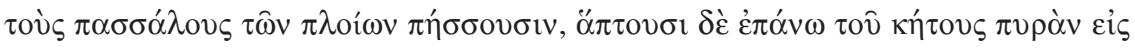

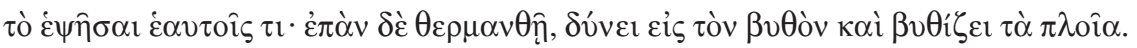

\section{${ }^{23}$ De principiis, IV.}

${ }^{24}$ Cf. Wellmann, 1930; Zambon, 2002, pp. 21-22.

25 "Bibbia, bestie e Bestiari: l'interpretazione cristiana degli animali dalle origini al Medioevo", in Scianchi, 1999, pp. 375-410, 396.

${ }^{26} \mathrm{Cf}$. Sbordone, 1936, p. 173. Per un approfondimento di questi concetti e le relazioni degli Alessandrini (partic. Filone, Clemente, Origene, Didimo) con i temi enunciati nel Physiologus, si rimanda a Grant, 1999, pp. 44-72. 


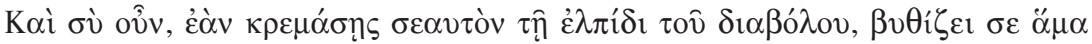

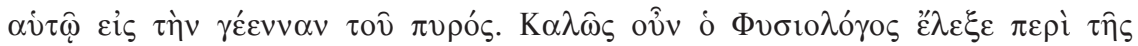

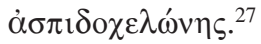

Così la storica traduzione italiana di Francesco Zambon, ${ }^{28}$ che qui riproduco per ovvi motivi di agevolezza:

Salomone 29 nei Proverbi esorta dicendo: "Non volgerti a una donna cattiva, perché stillano miele le labbra della meretrice, e a tempo opportuno essa unge la tua gola, ma in seguito la troverai più amara del fiele e aguzza più di una spada a due tagli. Poiché i piedi della follia conducono coloro che vi si affidano alla morte nell'Inferno". 30

C'è un mostro nel mare detto balena: ha due nature. La sua prima natura è questa: quando ha fame, apre la bocca, e dalla sua bocca esce ogni profumo di aromi, e lo sentono i pesci piccoli e accorrono a sciami nella sua bocca, ed esso li inghiotte; non mi risulta invece che i pesci grandi e adulti si avvicinino al mostro.

Così anche il demonio e gli eretici, con la seduzione e l'inganno, che sembra essere un soave profumo, adescano i piccoli e coloro che non hanno il senno adulto; quelli invece che hanno l'intelletto adulto, sanno di non poterli attrarre: tale fu Giobbe, tali Mosè, Isaia, Geremia, e tutta la schiera dei profeti; così Giuditta scampò ad Oloferne, Ester ad Artaserse, Susanna ai secchioni, Tecla a Tapiri.

L'altra natura del mostro: esso è di proporzioni enormi, simile a un'isola; ignorandolo, i naviganti legano ad esso le loro navi, come in un'isola, e vi piantano le ancore e gli arpioni; quindi vi fanno fuoco sopra per cuocersi

27 Physiologus Graecus, 17, ed. Sbordone, 1936, pp. 64-68. La presente edizione, sebbene non recente e non scevra di difetti, si distingue ancora oggi dalle altre per il gran pregio di basarsi sulla collazione di ben 77 manoscritti. Della cosiddetta "prima redazione", delle tre classificate da Sbordone, esistono 23 manoscritti datati tra i X e il XVII secolo; il più antico (fine X sec.) è il cod. 397 conservato alla Pierpont Morgant Library di New York ma proveniente da Grottaferrata (Cryptoferratensis, A 33), sconosciuto allo stesso Sbordone e ora pubblicato da Offermans, 1966.

${ }^{28}$ Zambon, 2002, pp. 56-57.

${ }^{29}$ Salomone, nome che significa "pacifico", nel Cantico è anche sponsus, è figura Christi. Su Salomone come profondo conoscitore e esegeta della natura in merito alla sua presenza nel Physiologus, vedi Faraci, 2003.

${ }^{30}$ Cf. Prov., 5, 3-5: Favus enim distillans labia meretricis, et nitidus oleo guttur eius; novissima autem illius amara quasi absynthium, et acuta quasi gladius biceps. Pedes eius descendunt in mortem, et ad inferos gressus illius penetrant. 
qualcosa: ma non appena esso sente caldo, s'immerge negli abissi marini e vi trascina le navi.

Se dunque anche tu, o uomo, ti tieni sospeso alla speranza del demonio, questi ti trascina con sé nella geenna del fuoco.

Bene dunque il Fisiologo ha detto della balena.

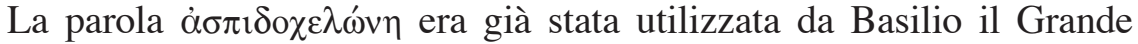
nel secolo IV e la si ritroverà cinque secoli dopo in Pietro di Sicilia. Nel primo, l'animale viene utilizzato come metafora dei rischi che possono occorrere ai confratelli qualora essi si lascino andare alle tentazioni delle donne e quindi alla intimità con esse. ${ }^{31}$ Ma Basilio evidentemente si rifà a quegli stessi versetti dei Proverbi richiamati dall'autore del Physiologus, benché il suo principale modello rimanga Ps 57, 4-6:

Alienati sunt peccatores a vulva; erraverunt ab utero, locuti sunt falsa. Furor illis secundum similitudinem serpentis, sicut aspidis surdae et obturantis aures suas, quae non exaudiet vocem incantantium, et venefici incantantis sapienter.

Nella Historia utilis et refutatio Manichaeorum vel Paolicianorum

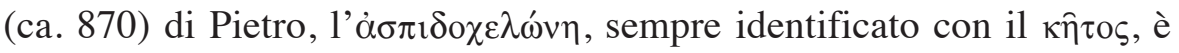
adoperato in funzione antiereticale. Pietro, difatti, attaglia a mo' di in-

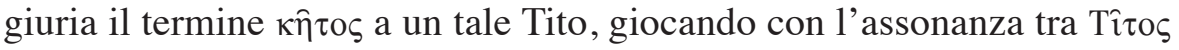

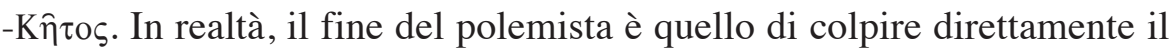
dualismo armeno dei Pauliciani attraverso colui che, come diretto continuatore del fondatore Costantino-Silvano (m. 682), ricopre il ruolo di secondo dei $\delta 1 \delta \alpha ́ \sigma \kappa \alpha \lambda o r$, vale a dire Simeone-Tito, attivo tra il 685 e il 688. ${ }^{32}$ Nell'ottica di Pietro, l'utilizzo della simbologia dell'isola-balena si rivela perfetta. Tito è perfido come il cetaceo, ne ha la stessa natura diabolica, e come il diavolo, egli attira a se stesso coloro che ignorano la sua malizia, trascinandoli con sé nel fuoco inestinguibile. ${ }^{33}$ La bestia

${ }^{31}$ Basilio, Sermo de contubernalibus (De Amicitia), PG 30, 824 C.

${ }^{32}$ Cf. Sfameni Gasparro, 1995, vol. 3, pp. 69-98, part. 73-79. Vedi anche Runciman, 1982, pp. 26-62 e 181; Obolensky, 1948, pp. 28-58; Lemerle-Redgate, 1998, pp. 193-194.

${ }^{33}$ L'abate Charles Auguste Auber ha giustamente commentato così questa allegoria: «Nous avons remarqué plusieurs fois que, le rôle principal de ce monstre étant celui du démon, les énormes proportions de sa gueule immense ont paru très-convenables à exprimer l'ouverture de l'enfer, si souvent reproduite pleine de flammes et d'âmes qui s'y engouffrent, jetées par les demons, ou qui en sortent appelées par le Fils de Dieu qui frappe de sa croix la gueule du Léviathan» (Auber, 1884, III, p. 499, n. 1). 
corrisponde in tutto a quella del Physiologus, fino a riprendere quel particolare meno comune, la voce, che ne contrassegna le versioni armena e siriaca. ${ }^{34} \mathrm{M}$. Canard dunque non si sbaglia quando ritiene che Pietro dovesse conoscere l'opera. ${ }^{35}$ Anzi, a mio avviso, si può anche immaginare che il racconto dell' isola-balena dovesse essere in qualche modo noto ai destinatari dello scritto anti-pauliciano e all'ambiente geografico nel quale essi erano attivi. Paradossalmente, il libretto alessandrino, erroneamente bollato come eretico, diventa strumento della lotta alle eresie cristiane. Ne diamo la traduzione latina presentata nella Patrologia, accompagnata tra parentesi, nei passaggi di maggior valore, dal corrispettivo testo greco edito da Denise Papachryssanthou: ${ }^{36}$

[...] Titus se nominavit. Ego vero hunc Titum: non enim imitator erat illius

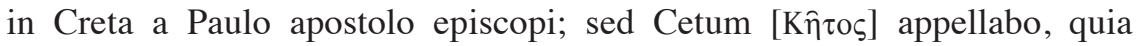

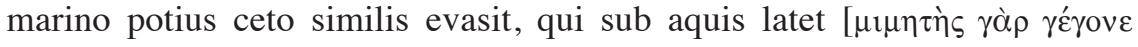

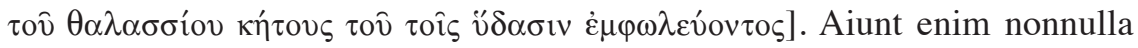

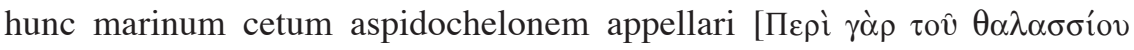

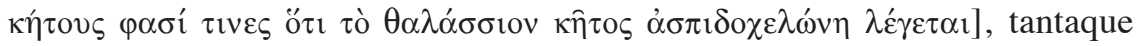

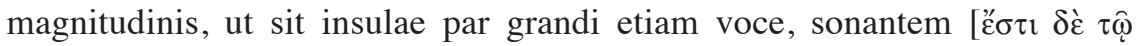

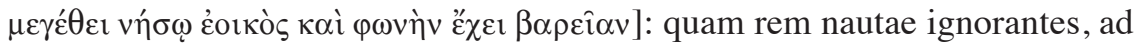
eum anchoras jaciunt clavisque confixis naves religant; ubi postquam focum [кó́ntvov] incenderit, calefactum animal repente se mergens, cunctos sub abisso suffocat. Sic iste eos qui immobilitatis ejus magnitudinem ignorabat, vocemque immanem non devitabant, sed potius auscultabant in coque spei

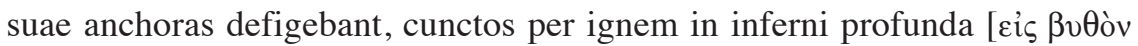

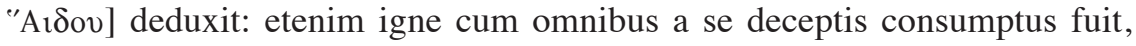
atque ad flammam migravit inexstinguibilem. ${ }^{37}$

Nel quadro del presente, specifico contesto dottrinale, a ragione $\mathrm{Ca}$ nard $^{38}$ ricorda il racconto De ceto et nauta piscatore contenuto nello Speculum sapientiae Beati Cyrilli (1. I, cap. 8), opera di dubbia autenticità e difficilmente databile. Canard pensa che autore ne fu Cirillo di

\footnotetext{
${ }^{34}$ Vedi più avanti.

${ }^{35}$ Canard, 1972, pp. 379-384.

${ }^{36}$ Papachryssanthou, 1970, p. 45 (in questa edizione il brano occupa i capitoli 107-109).

${ }^{37} \mathrm{PG}, 104,1282$.

${ }^{38}$ Canard, 1972, pp. 380-381.
} 
Alessandria, ma J. G. Th. Grässe ${ }^{39}$ editore del testo, la attribuisce a Cirillo di Quidenon, vissuto nel XIII sec. La trama è molto semplice: un pescatore approda su un'isola e vi accende un fuoco:

Super maris undam cetus grandissimus in insulam elevatus cum aridae (terrae) speciem mentiretur, piscator raticula fluctuans terrae cupidus in portum sophisticum hunc pervenit moxque laetus ibi descendens parum levata puppicula manu, petra et ferro genito rogo sibi pius coepit fovere corpusculum algore rigidum, labore fessum et esurie desiccatum.

Mossasi la balena, il pescatore le chiede perché avesse simulato l'immobilità, ma quella rigira la domanda all'uomo, accusandolo di aver riposto fiducia su una cosa mobile. Così, l'animale conclude invitando l'ingenuo a non credere nell'esistenza di ciò che appare alla vista; apparenza e realtà non coinciderebbero mai, sicché il sofista può sembrare un filosofo e l'ipocrita un santo: è folle colui che si affida a cose instabili o mobili e che non getta l'ancora su una terra solida. Il marinaio, spiritualmente edificato, può rimettersi in viaggio: ${ }^{40}$

Verum cum ignis acuta caliditas insensibilis piscis transacta pinguedine tandem in carnem sensibilem descendisset, ardoris sensu statim pisce commoto terrae motum aestimans nauta stupidus relictis omnibus cucurrit ad lignum. Sic inde veritate comperta tutus de barcula lamentabiliter coeto dixit: ut quid ostensa ingenti mole stabilitate letaliter simulata et cute picta mentiebaris te portum? Ni certe natabile lignum statim profugo subvenisset, in te confidentem raptus subito non minus quam naufragium submersisses. Cui piscis respondit: Tu autem quare ad quiescendum super rem mutabilem descendisti? Et ille: certe quia tellus esse et non aliud videbaris. Tunc alter: numquid omne quod videtur existit? An ignoras quod plura in sola sui apparentia, non in veritate fraudantur? Nam sophista videtur philosophus et hypocrita sanctus. Propter hoc, carissime, tu qui maris incola periculosis versaris in fluctibus et perniciei castris undique obsessus, in ligno perfragili circumdaris, quo nimirum in necessitate confugias, cui te credas, ac ubi stabilitus confidas, prius ruminante diligentia videas et clara prudentiae circumspectione attendas. Nam si ex toto semel erraveris, praeventus morte correctionis tibi postmodum nullatenus locus erit. Sic ergo tutum stabilitate perpetua quaerens portum, hunc meliorem et in bonis melioribus optimum

\footnotetext{
${ }^{39}$ Grässe, 1880, p. 13.

${ }^{40}$ Grässe, 1880, cf. Faraci, 1990, p. 155.
} 
prudenter eligas ubi confidentia secura quiescas. Nonne sapiens architectus in terra stabili fodiendo quadri fundamentum stabilius et navis pervigil gubernator, antequam suam accomodet ancoram, scrutator abyssi plumbata cordula quadri tutissimum fundamentum? Stultus enim est, qui super rem instabilem fabricat et amens ex toto, qui rebus mobilibus se ab eisdem stabiliendum commendat. Vade igitur et disce nequaquam in perituris confidere sed in terris de caetero tuae spem ancoram tantum fige. Quibus edoctus nauta consolatus discessit. ${ }^{41}$

È chiaro che nel Fisiologo l'ả $\sigma \pi 1 \delta 0 \chi \varepsilon \lambda \omega ́ v \eta$ è genericamente, e principalmente, figura diaboli, ma ad un livello interpretativo più profondo, esso diviene anche simbolo della moglie che tradisce il marito, della fornicazione e, più in genere, dei piaceri terreni più turpi. In particolare il parallelo della moglie adultera è recuperato, con esiti interessanti e originali, da Pier Damiani (1007-1772):

Sed dum de turpi infidelitate mulieris eloquimur, cetus etiam ille marinus nobis in memoriam revocatur qui nimirum super enorme corpus sabulum portat, ac terga sua super undas elevat, ut tanquam insula fluctus marinos excedat. Hanc igitur incunctancter insulam esse credentes remiges applicant, paxillos figunt, navim sistunt, focos instruunt, sed bellua mox ut ignis sentit ardorem, illico mergitur in profundum et navem simul ac nautas vorat ferale naufragium Nam et Gerardus, noster videlicet monachus, retulit in Normandiae littoribus captam se vidisse balaenam, de cujus linguae carnibus quatuordecim sagmarii sunt non leviter onerati. Sed ut ad aliud redeam unde digressus sum, quid mirum, si fidem in bellua nauta non reperit, quam et vir in uxore propria non invenit? Quid mirum, si bestia muta insulam mentita procellis immergitur, cum et mulier violata fide conjugii et ferinis amplexibus exponatur? Quid enim tam nostrum, et tam proprii juris in hoc mundo, cui varii casus possit deesse suspicio ${ }^{42}$

Dora Faraci ${ }^{43}$ ha escluso, e giustamente, l'assoluta dipendenza del noto episodio dell" "isola-balena" Jasconius ${ }^{44}$ della Navigatio sancti Brendani

\footnotetext{
${ }^{41}$ Grässe, 1880, pp. 13-14.

${ }^{42}$ De bono religiosi status et variarum animantium tropologia, XXX (De ceto), PL, 145,790 B-C.

${ }^{43}$ Faraci, 1991, p. 150 n. 3.

${ }^{44} \mathrm{Su}$ questo tema è in avanzato stato di preparazione un mio volume monografico.
} 
(VIII-IX sec.; cap. 10) ${ }^{45}$ dal più generico simbolismo dell' "isola galleggiante", meglio nota nella denominazione inglese di floating island. Il grande pesce della Navigatio è chiaramente altra cosa! E lo stesso pesce trasmetterà alcuni suoi aspetti e funzioni alle stesse versioni posteriori del Physiologus e dei vari bestiari. Sebbene la trama del racconto ad esso relativo si presenti quasi identica in talune immagini, bisogna rimarcare che dalla bocca di quello non fuoriesce il profumo che attira i piccoli pesci del Fisiologo, ovverosia la prima, o la seconda in altre versioni, delle due nature dell'aspidochelone. E con questo animale risultano poche, infine, le analogie oggettive. Jasconius, del resto, non è del tutto inatteso dalla ciurma: egli è rivelazione divina, provvidenziale. A Brendano il Signore tutto rivela, egli sa già, prima dei confratelli, di che creatura si tratti e perché la si incontri proprio in quel luogo, in un determinato momento. Il pesce non li inganna, ma anzi aiuta l'intera compagnia, volontariamente, offrendo loro un luogo dove pregare e pure dimorare, ma soprattutto celebrarvi la Pasqua, l'evento liturgico più importante della storia della Chiesa. Così il pesce farà per sette anni consecutivi. Fondamentale, essenziale meglio direi, diviene allora la funzione "sacrale" dell'animale, troppo specifica e densa di significati, evidentemente mai casuali, per ritenere siffatto episodio soltanto una trasposizione del

${ }^{45}$ Cum autem uenisset ad aliam insulam, cepit illa nauis stare antequam portum illis potuissente tenere. Sanctus Brendanus precepit fratribus exire de naui in mare, et ita fecerunt. Tenebantque nauim ex utraque parte cum funibus esque dum ad portum uenissente. Erat autem illa insula petrosa sine ulla herba. Silua rara erat ibi, et in litore illius nihil de arena fuit. Porro pernoctantibus in oracionibus et uigiliis fratribus foris, uir Dei sedebat intus in naui. Sciebat enim qualis erat illa insula insula, sed tamen noluit eis indicare, ne perterrerentur. Mane autem facto precepit sacerdotibus ut singuli missas cantarent, et ita fecerunt. Cum sanctus Brendanus et ipse cantasset [missam] in naui, ceperunt fratres carnes crudas portare foras de naui ut condirent sale [illas], et etiam pisces quos secum tulerunt de alia insula. Cum hoc fecissent, posuerunt cacabum super ignem. Cum autem ministrarent lignis ignem et feruere cepissent cacabus, cepit illa insula se mouere sicut unda. Fratres uero ceperunt currere ad nauim, deprecantes patrocinium sancti patris. At ille singulos per manus trahebat intus. Relictisque omnibus que portabant in illa insula, ceperunt nauigare. Porro illa insula ferebatur in oceanum. Potuti autem ignis ardens uideri super duo miliaria. Sanctus Brendanus narrauit fratribus quid hoc esset, dicens: "Fratres, admiramini quod fecit hec insula?" Aiunt: "Admiramur ualde nec non et ingens pauor penetrauit nos." Qui dixit illis: "Filioli, nolite expauescere. Deus enim reuelauit mihi hac nocte per uisionem sacramentum huis rei. Insula non est, ubi fuimus, sed piscis, prior omnium natancium in oceano. Querit semper ut suam caudam simul iungat capiti, et non potest pre longitudine. Qui habet nomen Jasconius" (Selmer, 1959, pp. 20-21). 
"modello fisiologico", $\mathrm{o}$, ancor più erroneamente, uno strumento di inganno in una banale variante dell'aspidochelone del Fisiologo, del quale il cetaceo non sarebbe altro che un mero "symbole de la fausse apparence". ${ }^{46} \mathrm{Si}$ aggiunga che i confratelli che accompagnano san Brendano non sono ritenuti dei peccatori, a differenza dei navigatori presentatici dal Fisiologo. Pertanto, Jasconius non ha il compito di trascinarli in acqua, e l'anno seguente, fatto singolare, sul suo dorso è rinvenuta la pentola lì abbandonata e già vista ancora al primo anno sul pesce fino a una distanza di due miglia dal curach. Jasconius è creatura mansueta, ${ }^{47}$ sottomessa, paziente, come fa ancor più intendere lo stesso santo: Considerate quomodo subiugat Deus inmanissimam bestiam subtus nos sine ullo impedimento $(15,38-39)$.

Nella descrizione dell'aspidochelone si pone l'accento sulla sabbia che copre il dorso, la prima delle ragioni per cui i marinai la credono isola. La crescita della vegetazione potrebbe essere il risultato dell'accumulo di sabbia, ma non può certo escludersene la funzione strumentale all'inganno. Eppure, la vegetazione non è mai menzionata nella presente redazione, né nella latina, al di là della versione greca contenuta in un tardo codice di Smirne distrutto dai Turchi nel 1922. ${ }^{48}$ Tuttavia, anche qui viene mostrato un pesce con una barca su di esso, una sorta di piattaforma di terra con una coppia di alberi e due marinai presso un fuoco. ${ }^{49} \mathrm{Ma}$, in ogni caso, non si posseggono elementi validi per determinare se il motivo dell'albero provenisse da un più antico Fisiologo o se si trattasse soltanto di una tarda aggiunta.

Nel suo Hexaemeron, il vescovo antiocheno Eustazio, morto nel

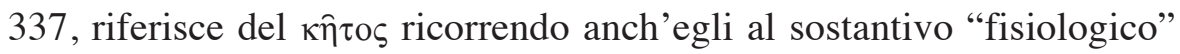
'A $\pi \iota \delta о \chi \varepsilon \lambda \omega ́ v \eta$. Egli ne parla come di un animale molto grande, con un corpo simile a roccia liscia, avvicinato dai marinai poiché creduto isola. Così, dopo che quelli vi fissano dei pali per l'ormeggio, il pesce, pure a causa del riscaldamento causato dalla lunga esposizione al sole, emettendo una voce oltremodo grave, ${ }^{50}$ ritorna nell'abisso:

46 James-Raoul, 2002, p. 215.

${ }^{47}$ Così già notava l'imprescindibile articolo di Carp, 1984, p. 134.

${ }^{48}$ Cf. Faraci, 1991, p. 155 n. 14.

${ }^{49}$ Vedi Demus, 1976, pp. 232-257.

${ }^{50}$ Certamente un riflesso del canto maliardo, soave ma mortifero, delle sirene, la cui natura allegoricamente "diabolica", insieme a quella molto simile degli ippocentauri, 


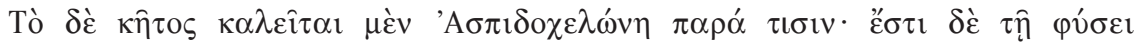

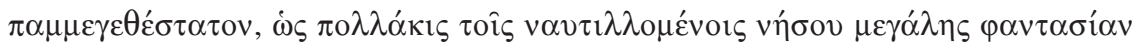

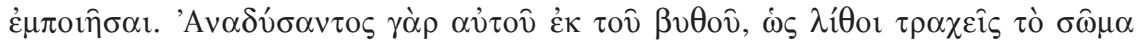

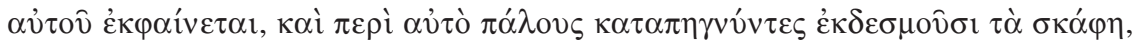

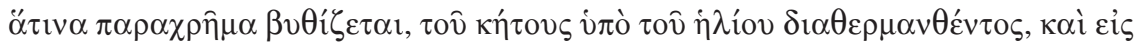

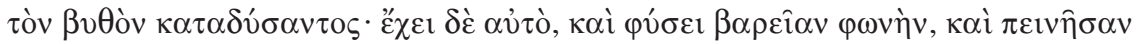

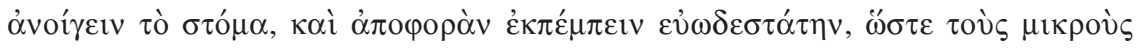

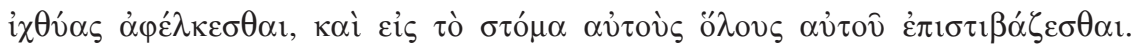

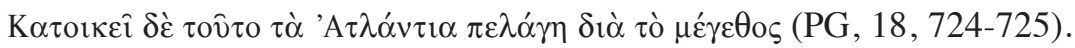

Questo racconto, in entrambe le nature, concorda chiaramente con quanto riferito nelle versioni orientali del Physiologus, anche se rimane singolare la scomparsa del fuoco, qui sostituito dal calore del sole. Si tratta

era già trattata nel Fisiologo greco, "ha detto il profeta Isaia: 'Gli spettri e le sirene e i ricci danzeranno in Babilonia"” [Is. 13, 21]. Il Fisiologo ha detto delle sirene e degli ippocentauri: ci sono nel mare degli animali detti sirene, che simili a muse cantano armoniosamente con le loro voci, e i naviganti che passano di là quando odono il loro canto si gettano nel mare e periscono. Per metà del loro corpo, fino all'ombelico, hanno forma umana, per la restante metà, d'oca (cf. Apollonio, Argonautica, IV 898-899: “apparivano in parte simili a fanciulle nel corpo, e in parte ad uccelli", trad. A. Borgogno, Milano, 2003; Isidoro, Etymologiae, XI, 3, 30: Sirenas tres fingunt fuisse ex parte virgines, ex parte volucres, habientes alas et ungulas) [...] Così anche ogni uomo indeciso, incostante in tutti i suoi disegni. Ci sono alcuni che si radunano in Chiesa e hanno le apparenze della pietà, ma rinnegano ciò che ne è la forza, e in Chiesa sono come uomini, quando invece se ne allontanano, si mutano in bestie. Costoro sono simili alle sirene e agli ippocentauri: infatti " "con le loro parole dolci e seducenti', come le sirene, 'ingannano i cuori dei semplici' [Rom. 16, 18] ...” (cap. XIII, trad. Zambon, 2002, pp. 52-53). Il capitolo corrispondente della versione armena è il XV. Sulle "sirene alate", precedenti nel tempo quelle "con la coda di pesce" (cf. Liber monstrorum, I, 6: Sirenae sunt marinae puellae quae navigantes pulcherrima forma et cantu mulcendo decipiunt et a capite usque ad umbilicum sunt corpore virginali et humano generi simillimae; squamosas tamen caudas habent, quibus sempre in gurgite latent, ed. F. Porsia, Bari, 1976, p. 149), vedi Cattabiani, 2001, pp. 501-511. In Agostino gli ippocentauri simboleggiano i Manichei (Contra Faustum, XV, 9, CSEL, 25, p. 436, 11. 2-6: Quem dicis hippocentaurum, nesciens quid loquaris, nec adtendis, quid tibi tua fabula confecerit, cum ex parte dei tui et ex parte terrae tenebrarum falsum mundum fabricat in corde tuo. Ita ne ille non est hippocentaurus semiferus et semideus? Uere, quia nec hippocentaurus dicendus est). Cf. Liber monstrorum, I, 7: Hippocentauri equorum et hominum commixtam naturam habent et more ferarum sunt capite segoso, sed ex parte aliqua humanae normae simillimo quo possunt incidere loqui: sed insulta labia humanae locuzioni nullam vocem in verba distinguunt (ed. Porsia, op. cit., p. 150). Sulla sopravvivenza e le varie riletture del mito delle sirene nell' antichità cristiana è necessario vedere Rahner, 1990, pp. 383-402. 
di un particolare però non nuovo. Già Claudio Eliano (II-III d. C.), infatti, valente erudito in tema di questioni zoologiche, rilevava l'abitudine di questo cetaceo di rimanere in superficie allo scopo di riscaldarsi ai raggi

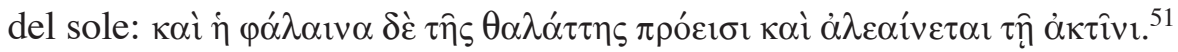
Si badi, tuttavia, che il celebre sofista utilizza altrove il più comune

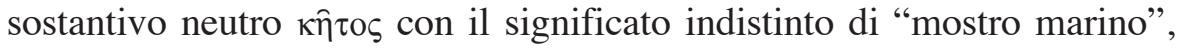
come quando, ad esempio, riferendo della pesca delle genti dell'isola di Taprobane, sottolinea la distinzione tra i "pesci" in genere e i кń $18,19)$; di contro, nell'elencare i cetacei tanto diffusi nell'Oceano Indiano, ${ }^{52}$ egli impiega il più specifico qú $^{\prime} \alpha \iota v \alpha$ (XVI, 18,41).

\section{Le versioni orientali}

Enrico Cerulli ${ }^{53}$ ha isolato alcuni passaggi, rilevanti in questa sede, all'interno della raccolta dei Miracoli operati dal santo egiziano Giulio di Aqfahs, ${ }^{54}$ tradotti dall'arabo in etiopico, riuniti in'unica omelia attribuita erroneamente al patriarca copto Alessandro I (inizi IV sec.), ma redatti, o legati, tra XI e XIV sec. ${ }^{55}$ Giulio visse in età dioclezianea,

${ }^{51}$ De natura animalium, IX, 50, 5-6. Specificamente alla $\varphi \alpha ́ \lambda \alpha \iota v \alpha$ Eliano menziona inoltre il caratteristico sfiatatoio e il colore bianco (II, 525-526), il quale, però, potrebbe qui indicare una probabile confusione dell'autore con un altro cetaceo, il beluga, sorta di "delfino bianco", detto essere proprio "bianco come la neve" (cf. Delort, 1993, p. 35).

${ }^{52}$ Cf. Liber monstrorum, II, 26 (De ballaena): Ballaena quoque intolerabilis fera in India nascitur, ubi plurima prope totius orbis prodigia leguntur; de quorum pellibus beluarum sibi gens quaedam apud Indos vestimentorum termina componit (ed. F. Porsia, op. cit., p. 248). Questo celebre testo, opera di un anglosassone dell'vIII secolo forse istruitosi anche in Irlanda, è una preziosa summa di leggende teratologiche e di orientalia mirabilia estratti perlopiù dagli autori latini, con particolare attenzione a Virgilio, Ovidio e Lucano, da quella tradizione della storiografia romanzata che da Ctesia passa per lo Pseudo-Callistene e culmina nei gesta Alexandri di età altomedievale, oltreché, e non in minor misura, dai Padri, Agostino, Girolamo e Isidoro in testa, e, ma solo occasionalmente, dal Physiologus (vedi il denso ed esaustivo studio introduttivo di Franco Porsia all'edizione citata, particolarmente pp. 9-41 e 63 ss.).

${ }^{53}$ Cerulli, 1946.

${ }^{54}$ Ed. e trad. in Cerulli, 1959.

${ }^{55}$ I Miracoli di Giulio sono venti racconti dalla diversa origine successivamente legati, per cui non possiamo stabilire una precisa datazione, ma solo quella, appunto, di quando essi furono legati. 
fu carceriere pietoso in Egitto negli anni della persecuzione, ascoltava i resoconti dei martiri ed è, per tradizione, autore di Atti; infine si convertì e, divenne amico dei martiri, per poi farsi martire egli medesimo.

Nel terzo racconto dei Miracoli si parla di un'isola che "cammina" nel Mare Egeo e minaccia di affondare con molti dei passeggeri di una nave sbarcati su quella; in realtà si tratta di una balena, alla quale Giulio, apparendo, ordina di riportare gli uomini e le loro navi, a rimorchio, fino al porto di Alessandria. L'episodio dei Miracoli di Giulio è fondamentale, unico, perché il salvataggio è il momento cruciale e distintivo. Il motivo della "balena prodigiosa" o salvatrice ritorna anche nell'opera etiopica Ta'amra Māryām, ovvero i Miracoli di Maria: in un monastero copto dei monaci hanno per dodici anni al loro servizio un diavolo che un giorno porta nel convento una balena sulla quale è una nave con molti naviganti, che così si convertono e si fanno monaci; la nave è posta «sopra quel pesce, gran cetaceo», dice il testo. ${ }^{56}$

A partire dai secoli v e VI il Fisiologo inizia a diffondersi capillarmente, com'è attestato dalle traduzioni in etiopico, siriaco, armeno, arabo e latino.

La più antica delle versioni orientali è quella etiopica, strettamente collegata alla redazione greca più antica e risalente ai primi decenni del V secolo. ${ }^{57}$ Pervenutaci attraverso tre manoscritti del XV secolo, è formata da 48 capitoli, secondo il contenuto e la sequenza del codice A, un parigino del XV secolo (graecus 2426), edito da Pitra. ${ }^{58}$ Carlo Conti Rossini ne ha curato edizione critica e traduzione italiana: ${ }^{59}$

A proposito dell'aspadakloni che è il capo delle bestie della terra. Il suo corpo è forato ${ }^{60}$ Salomone dice nei proverbi $(5,34)$, mentre insegna e dimostra: Non guardare la donna cattiva, perché miele scorre dalle labbra della donna fornicatrice; ma dopo troverai ciò che è amaro più del fiele, ed affilato assai più d'un rasoio dal doppio taglio. In ugual modo, adunque, nel mare vi è

56 Trad. Cerulli, 1946, p. 447, n. 3. Cf. Cerulli, 1943.

${ }^{57}$ Cf. Dolcetti Corazza, 1992, pp. 27 ss. Edizione critica e traduzione tedesca in Hommel, 1877; lo stesso studioso proporrà pochi anni dopo una più accurata traduzione in Hommel, 1890, pp. 13-36.

${ }^{58}$ Pitra, 1965, III, pp. 338-373.

${ }^{59}$ Conti, 1951.

${ }^{60} \mathrm{Il}$ curatore crede che qui l'anonimo traduttore volesse intendere il foro caudale del guscio della tartaruga (p. 29, n. 5). 
un cetaceo che si chiama aspadakloni, che ha doppia natura: quando ha fame, spalanca la sua bocca, e i pesciolini ne aspirano il profumo, ed esso li inghiotte quando siensi radunati; ma i pesci grossi non si accostano a quel cetaceo. - In tale senso parlano Giobbe, Mosè, Geremia e tutta l'accolta dei Profeti. Giuditta, a sua volta, mise in fuga Oloferne e lo vinse; ed altrettanto fece Ester ad Artaserse, Susanna ai vecchissimi, Tecla a Tamrenes. Seconda natura del cetaceo. Esso è grosso quanto un'isola. Non conoscendolo, coloro che fanno arrostire i pesci si comportano su quel cetaceo come se fossero in un'isola: per il calore, esso sommerge in mare e fa naufragare le navi.- Se tu pure ti pianti su lui, il diavolo si rallegra, e ti fa calare seco nell'abisso della gehenna. Giustamente ha detto il Fisiologo (XVII, pp. 29-30).

Nell'edizione del testo etiopico curata da Budge, diversamente dalla redazione greca e dalla siriaca, la balena inghiotte i naviganti: "ordinai loro di salire sulla nave; e [Filone] pose con loro molte armi, marine e terrestri. E mandò con loro 800 uomini dei grandi del regno. Quando si avvicinarono ad essa (isola) e la videro, ecco una fiera marina inghiottì la nave e quel che era sopra". ${ }^{61}$

Secondo Cerulli, ${ }^{62}$ nel Physiologus si tratterebbe davvero di un'isolatartaruga, e non di una balena o grande pesce alla stregua dell" "isolabalena" Jasconius, che del resto è "veicolo di salvezza", "nuovo carro senza veli né remi", ${ }^{63}$ per quanto le due cose siano sovrapposte. Nella versione etiopica del Physiologus nell'edizione di F. Hommel, tradotta sempre da Cerulli, le differenze dalla greca rientrano: "Essa è molto grande come un'isola. Ed, ignorando [i naviganti], si pongono su quel cetaceo come su di un'isola a cuocere pesce. Pel calore (del fuoco) si immerge nel mare ed affonda così anche le navi". ${ }^{64}$

Contemporaneo dell'etiopico è il siriaco, in principio pubblicato, insieme alla traduzione latina, da O. G. Tychsen nel $1795 .{ }^{65} \mathrm{Il}$ documento si discosta da tutti gli altri per la mancanza di commenti religiosi e di insegnamenti morali o dottrinari, limitandosi a una trattazione di tipo naturalistico molto fedele all'originale che manifesta gli intenti scientifici del traduttore. Quasi un secolo dopo ne sarà invece edita una versio-

\footnotetext{
${ }^{61}$ Trad. Cerulli della edizione etiopica di Budge, Cerulli, 1946, p. 446, n. 1.

${ }^{62}$ Cerulli, 1946, p. 447.

${ }^{63}$ Cerulli, 1946, p. 448, n. 1.

${ }^{64}$ Cerulli, 1946, p. 447, n. 1.

65 Tychsen, 1795.
} 
ne più recente, nota come Leidensis, contenente un particolare singolare già letto in Eustazio e sconosciuto ad altre versioni dell'opera. Si tratta, di fatto, di una versione contenuta in un manoscritto del secolo XI, perlopiù rifacentesi alla redazione greca originale di 47 paragrafi, ma con l'aggiunta di capitoli desunti dall'Hexaemeron di Basilio: ${ }^{66}$

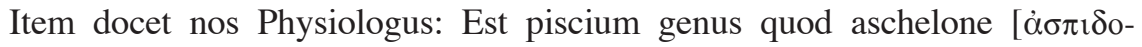
$\chi \varepsilon \lambda \omega ́ v \eta]$ vocatur. Hic piscis ingens est veluti insula, et vox ejus terribilis et gravissima. Et nautae ei nondum assueti et ejus ignari subinde tergum ejus conspiciunt et insulam esse opinati eum ascendunt, navem alligant et ignem in eo accendunt. Quum autem igne calescit et aduritur, in mare profundum se demittit et etiam illos demergit qui in eo versantur et portantur.

Theoria. Sic etiam nos ubi non exploramus et perspicimus aliquam (opinionem) veram esse et hanc amplexamur eique navem animae nostrae i. e. fidem nostram alligamus, perimus et demergimur, quum nobis obviam facta sit haeresis aliqua cui confiderimus et navem alligaverimus, veritatem ea teneri judicantes. ${ }^{67}$

Land, ancora, editò per primo la versione araba, fornendone pure una traduzione latina, ${ }^{68}$ risalente alla prima metà del $\mathrm{v}$ sec. e considerata molto importante giacché strettamente dipendente da quella greca più antica. Il testo è molto libero e si aggiungono nuovi particolari, forse anche attraverso una verosimile mediazione siriaca:

${ }^{66}$ Cf. Dolcetti Corazza, 1992, p. 32. Nel 1959 A. van Lantschoot ha pubblicato e tradotto dei frammenti in siriaco da un codice vaticano (syr. 555) che contengono anche il brano della balena (pp. 46-47): “Au sujet de la baleine. Le Physiologus nous apprend qu'il esiste une espèce de poissons dans la mer dénommée ỏ $\sigma \pi \imath \delta$ ¿ $\varepsilon \lambda \omega ́ v \eta$, (poisson) très grand (offrant) l'aspect d'îles; sa voix est terrible et très profonde. Les marins, ceux qui ne le connaissent pas, lorsq'ils voient son dos, pensent qu'il est une île ; ils débarquent, amarrent leurs vaisseaux et allument un feu sur lui. Quand il sent la chaleur et s'échauffe par suite du feu, il plonge dans la mer, immergeant aussi ceux qui sont étendus confiants sur lui. Ainsi de même nous. Si nous n'examinons et ne voyons pas ce qui est vrai, ne nous convertissons et n'appréhendons pas en esprit (le vrai), et en cela amarrons le vaisseau de notre âme, ce qui est notre foi, nous périssons et sommes immergés, car une des hérésies a pris possession de nous, nous avons eu confiance en elle et avons lié à elle le vaisseau de notre âme alors que nous pensions appréhender le vrai" [continua con la seconda natura].

${ }^{67}$ Ed. Land, 1875, pp. 31-98, trad. pp. 86-87.

${ }^{68}$ Land, 1875, p. 174. Recentissima, ma a me inaccessibile, è l'edizione accompagnata dalla traduzione in lingua tedesca curata da Wentker, 2004. 
Est belva in mari, quae ubi esurit os aperit, unde prodit odor omnibus odoribus suavior. [...] et est illi natura altera. Ipsa enim speciem praebet insulae in medio mari; et veniunt naves et ad eam appellunt; et paxillos ei infingunt quibus naves alligant. Atque illa haec patitur donec nautae ignem accendant, et ubi sentit ignis ardorem, descendit ad infima terrae et maris fundum, et simul perdit omnes quae super ea sunt et naves et creaturas. -Itaque tu homo nolit cupida esse anima tua earum quae a Satana oriantur rerum bonarum, neve hujus dictis obsequi neve seduci te pati illius operibus, ne te submergat sicut submergitur navis cum omnibus quae in ea sunt, sed Deo confidito, qui te liberabit. ${ }^{69}$

Il Physiologus armeno, edito in principio dal Pitra, ${ }^{70}$ è comunemente ascritto ai secoli VI-VII. Studi più recenti, tuttavia, dimostrano che non sarebbe peregrino datare agli anni precedenti il $470^{71}$ quella traduzione verosimilmente effettuata su una redazione greca che Pitra medesimo ascrive al IV secolo. Si tratta, in ogni caso, di una versione che presenta due peculiarità non di poco conto: il soggiorno della bestia in luoghi sabbiosi e, come nella redazione siriaca, la sua capacità di emettere terribili suoni: Physiologus dicit: Est animal in mari, cui nomen Vanagria, marina testudo [scutifera testudo], quod aequale est balaenae; est in locis arenosis, similis insulae; vox ejus veluti belluae saevissimae $[. ..] .^{72}$ Il testo integrale dell'opera è ora disponibile nella nuova edizione con traduzione inglese di Gohar Muradyan. Così il brano dedicato alla "balena", canonicamente incastonato tra la natura della pantera e della pernice al capitolo XX:

The Apostle Paul says that "we are not ignorant of his design" $(2$ Cor. 2, 11) and that "he goes every way that is not good" (Ps. 36, 4). Physiologus says: there is a beast in the sea that is called shield-turtle. The dragon-fish is like a sandy island; its voice is of a harsh-voiced beast. And sailors being ignorant, having reached tie themselves as to an island, stick in pales and cast anchors. And when they light fire above, the place becomes warm, and plunges into the abyss and sinks many ships that are tied to it. And you, if you tie your hope to the devil, he will sink you to the Gehenna of fire [segue la seconda natura]. ${ }^{73}$

${ }^{69}$ Vedi Lauchert, 1974, pp. 79 ss.; cf. pure Dolcetti Corazza, 1992, p. 36.

70 Spicilegium Solesmense..., III, pp. 374-390.

${ }^{71}$ Cf. Muradyan, 2005, pp. 81-82.

72 Spicilegium Solesmense..., III, p. 383. Una traduzione inglese dell'intero documento è in Muradyan, 2005, pp. 141-162.

${ }^{73}$ Muradyan, 2005, pp. 153-154. 


\section{Le traduzioni latine e il Bestiario medievale}

Stabilire a che periodo esatto risalga la prima traduzione latina del Physiologus non è impresa facile. Se in un primo momento si era ipotizzato la fine del IV secolo ${ }^{74}$ indagini più attente hanno rimescolato le carte, così che l'unica datazione assolutamente certa rimanga ad oggi quella dei più antichi manoscritti di questo testo, risalenti al secolo VIII. ${ }^{75}$ L'importanza della traduzione latina è tale che ad essa sono informate tutte le versioni occidentali medievali, e in particolar modo i testi di area anglosassone,$^{76}$ anche se permane l'annosa difficoltà di stabilire su quale redazione greca sia stata effettuata la prima traduzione latina. ${ }^{77}$

In principio le versioni del Physiologus erano ritenute essere tre (A, $\mathrm{B}, \mathrm{C}){ }^{78}$ ma successivamente se ne contò una quarta, la $\mathrm{Y}$, non meno importante delle altre. Di queste, la $\mathrm{C}$, formata da soli 26 capitoli, è considerata, insieme alla Y, l'unica originaria e autonoma, nonché la più antica (sec. VI). ${ }^{79}$ Tuttavia, il codice più antico che contenga una versione latina del Physiologus risale al secolo VIII (ms. Berna, Bürgerbibliothek Lat. 611), redatto nella Francia orientale, in territori dunque carolingi ${ }^{80}$ In un primo momento edito diplomaticamente da Francesco Sbordone,${ }^{81}$ oggi è confluito nell'edizione della versio Y curata da Francis J. Carmody, il quale, insieme al suddetto, ha collazionato due codici monacensi rispettivamente del IX e del X secolo. Questa versione, che insieme alla $C$ è l'unica "originaria", 82 è in verità la più vicina all'originale greco ${ }^{83}$ ed è formata da 49 capitoli, con la balena ancora

${ }^{74}$ Carmody, 1938, p. 153, n. 3; Carmody, 1939, pp. 7 ss.

${ }^{75}$ Cf. Orlandi, 1985, p. 1068.

76 Cf. Faraci, 1990, pp. 88-118. Sull'eredità fisiologica del simbolismo animale altomedievale, vedi anche Voisenet, 1994, pp. 106-114, 257-319.

${ }^{77}$ Cf. Sbordone, 1949. Vedi anche, ma più in generale, Dolcetti Corazza, 1992, p. 49.

${ }^{78}$ Edite tutte insieme da Cahier-Martin, 1847-1856.

${ }^{79}$ Cf. Carmody, 1944; Orlandi, 1985, pp. 1087-1089; Dolcetti Corazza, 1992, p. 56.

${ }^{80}$ Cf. Cometta, 2004, p. 7.

${ }^{81}$ Sbordone, 1944, pp. 89-117.

${ }^{82}$ Cf. Orlandi, 1985, pp. 1082 ss.

${ }^{83}$ Vedi Henkel, 1976. 
preceduta e seguita rispettivamente dalla pantera e dalla pernice, ma i capitoli qui interessati sono solamente quelli dal 29 al 31. La descrizione e i caratteri dell'animale sono identici a quelli riportati nel testo greco, eccezion fatta per l'aggiunta della sabbia sul dorso:

Physiologus autem dixit de ceto quoddam, quod est in mari, nomine aspidoceleon uocatur, magnum nimis, simile insule, et plus quam harena gravis, figuram habens diabuli. Ignorantes autem naute, alligant ad eum naues sicut ad insulam, et anchoras et palos nauis configunt in eo; et accendunt super ignem ad coquendum sibi aliquid; si autem excaluerit cetus, urinat, descendens in profundum, et demergit omnes naves. Sic et tu, o homo, si suspendas te et alias te ipsum in spe diabuli, demergit te secum simul in gehennam ignis.

Aliut naturale habet cetus: si autem esurierit, multum adaperit os suum, et omnis odor bonus per os eius procedit; odorantes autem pusilli pisciculi, secuntur eius odorem, et componant se in ore magni ceti illius; cum autem impletum fuerit os eius, concludit os suum, et gluttit pusillos omnes illos pisciculos, hoc est modicos in fide. Maiores autem et perfectos pisces non inuenimus adpropiare ad cetum: consummati enim sunt perfecti; etenim Paulus apostolus dixit: Non enim eius uersutias ignoramus [2 Cor., 2, 11]. Iob perfectissimus piscis est, Moises et reliqui alii prophete; Ioseph effugit cetum magnum, principis cocorum mulierem, sicut in Genesis scriptum est [Gen., 39]; sicut et Thecla Thamyridum, sicut Susanna duos senes Babylonicos iniquos [Dan., 13]; Hester et Iudit effugerunt Artaxersen et Olofernem [Idt., 13]; tres pueri nabuchodonosor regem, magnum cetum [Dan., 3]; et Sara filia raguelis Nasmodeum (sicut in Tobia [Tob., 3, 7-17]). Bene ergo Physiologus dixit de aspidoceleon ceto magno. ${ }^{84}$

In codici carolingi è pure conservata la versio $\mathrm{B}$ (37 capitoli), la più diffusa nell'Occidente medievale a partire dal Mille.$^{85} \mathrm{Il}$ codice più antico è il Lat. 233 della Biblioteca civica di Berna, originario della regione della Loira e risalente al IX secolo. Il testo denuncia una notevole indipendenza rispetto al modello greco, ${ }^{86}$ sebbene nulla di particolarmente originale si aggiunga alle caratteristiche della balena, che qui occupa la posizione 24 :

Est belua in mare quae dicitur graece aspidochelone, latine autem aspido testudo; cetus ergo est magnus, habens super corium suum tamquam sabulones, sicut iuxta littora maris. Haec in medio pelago eleuat dorsum suum

${ }^{84}$ Carmody, 1941, p. 125.

${ }^{85}$ Cf. Orlandi, 1985, p. 1077, e Morini, 1996, pp. XII ss.

${ }^{86}$ Cf. Orlandi, 1985, p. 1077, e Dolcetti Corazza, 1992, pp. 52-53. 
super undas maris sursum; ita ut nauigantibus nautis non aliud credatur esse quam insula, praecipue cum uiderint totum locum illum sicut in omnibus littoribus maris sabulonibus esse repletum. Putantes autem insulam esse, applicant nauem suam iuxta eam, et descendentes figunt palos et alligant naues; deinde ut coquant sibi cibos post laborem, faciunt ibi focos super arenam quasi super terram; illa uero belua, cum senserit ardorem ignis, subito mergit se in aquam, et nauem secum trahit in profundum maris. Sic patiuntur omnes qui increduli sunt et quicumque ignorant diaboli astutias, spem suam ponentes in eum; et operibus eius se obligantes, simul merguntur cum illo in gehennam ignis ardentis: ita astutia eius $[\ldots]{ }^{87}$

Successive al x secolo risultano le altre versioni latine e i Bestiari, che del Physiologus latino sono il primo e più autentico frutto.

Da una "brutale giustapposizione" 88 di elementi della versio $B$ e delle Etymologiae di Isidoro (560 ca.-636) nasce quella che non a caso è detta versio B-Is ${ }^{89}$ pervenutaci in codici non anteriori alla fine del sec. XI (ms. Vaticano Pal. Lat. 1074), ${ }^{90}$ e punto di partenza quasi esclusivo per i successivi rimaneggiamenti e per le traduzioni vernacolari, in particolare quelle antico-francesi del XII e XIII secolo. ${ }^{91} \mathrm{Al}$ XXV capitolo, ${ }^{92}$ tra la pantera e la pernice, si tratta della balena alla sezione denominata Cetus, et quomodo decipiuntur naves ab eo. L'equazione "cetaceo = diavolo"

${ }^{87}$ Carmody, 1939, p. 44. La versio $C$ è una traduzione molto fedele del Physiologus greco: Est cactus [sic] in mare, aspido helune: duas naturas habet. Prima haec est: [...]. De natura seconda piscis. Quia talis tot exhibeat ei in modico insule [sic]. Navigantes autem suspicantur insulam esse; et post flagitium tempestatis ligent naves suas ad eam. Et dum accenderint ignem super eam ad coquendum aut calefaciendum, et si (ista?) sentiens ignem demergit se in profundum et trahit post se omnes naves ligatas. Huic animal [sic] similabitur mulieres fornicariae: de qua [sic] dicit Salomon (Prov. V, 3-5): "Mel enim stillat de labiis meretricis, quae proscurio unguet [sic] tuas fauces, postea autem amarius [sic] felle invenitur, et cautior (acutior?) magis quam gladius bis acutus, qui [sic] demergit in tenebris [sic] peccatorem". Perfecti autem et cauti non adpropinquant ei: qualis erat Ioseph apud mulierem aegyptiam, qualis erat Helias Zezabel [sic] arguens, qualis erat Susanna in medio seniorum. Bene ergo simulata (similata) est mulier huic pisce [sic] (ed. Cahier-Martin, 1847-1856, III, p. 253).

${ }^{88}$ Così Orlandi, 1985, p. 1094.

${ }^{89}$ Cf. McCulloch, 1962, pp. 28-30.

${ }^{90}$ Questo codice è tradizionalmente datato al x secolo, ma Orlandi lo crede più tardo (Orlandi, 1985, p. 1095).

${ }^{91}$ Cf. Orlandi, 1985, p. 1095, e Morini, 1996, p. 5.

92 Testo e traduzione italiana a cura di Morini, 1996, pp. 60-63. 
trova un sostegno più forte nelle considerazioni finali isodoriane, tese a ridurre il cetaceo alla stessa creatura infera che inghiotte Giona:

(Etymologiae, XII, 6, 7-8: Ballenae autem sunt inmensae magnitudinis bestiae, ab emittendo et fundendo aquas vocatae; ceteris enim bestiis maris

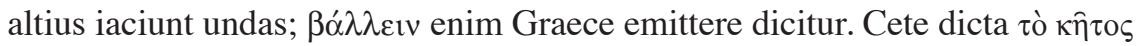

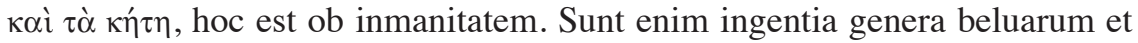
aequalia montium corpora; qualis cetus excepit Ionam, cuius alvus tantae magnitudinis fuit ut instar obtineret inferni, dicente Propheta: «Exaudivit me de ventre inferni [Ion., 2, 3].»)

Est belua in mari que dicitur grece aspidochelone, latine autem aspido testudo. Cetus ergo est magnus, habens super corium suum tanquam sabulum quod est iuxta litus maris. Hec in medio pelagi elevat dorsum suum per undas maris sursum, ita ut navigantibus nautis non aliud credatur esse quam insula, precipue cum viderint totum illum locum sicut in omnibus litoribus maris sabulo esse repletum. Putantes autem insulam esse, applicantes navem suam iuxta eam et descendentes, figunt palos et alligant naves. Deinde ut coquant sibi cibos post laborem, faciunt ibi focos super arenam quasi super terram. Illa vero, ut senserit ardorem ignis, subito mergit se in aqua et navem secum trahit in profundum maris. Sic paciuntur omnes qui increduli sunt et quicumque ignorat diaboli astucias, spem suam ponentes in eum ; et operibus eius se obligantes, simul merguntur cum illo in gehennam ignis ardentis: ita est astucia ejus [...].

Dopo l'esposizione della seconda natura, chiude così il passo di Isidoro:

Ethim[ologia] Cetus dicitur ob immanitatem corporis. Sunt enim ingentia corpora et genera beluarum, equalia montibus in tantum, ut eciam ibi naves quasi ad insulam applicentur, sicut ille qui accepit Ionam. Cuius alvus tante magnitudinis fuit, ut putaretur infernus, dicente ipso Iona propheta: "Exaudivit me de ventre inferi" [Ion., 2, 3]. ${ }^{93}$

A questa versione latina si ispirerà il poeta anglo-normanno Philippe de Thaun per il suo rifacimento in 3194 versi, scritto in Inghilterra tra il 1121 e il 1135; in realtà, malgrado il suo autore lo classifichi come Bestiario, quest'opera risulta essere un Physiologus tripartito nella materia

\footnotetext{
${ }^{93}$ Non v'è dubbio che tra le principali fonti del dodicesimo libro delle Etymologiae (De animalibus), interamente dedicato agli animali, vi fosse anche il Physiologus (cf. Brehaut, 1912, pp. 143 ss.).
} 
tradizionale (de bestiis, de avibus, de lapidibus). Come già accadeva per la versio B-Is, la balena, collocata al capitolo 23, laddove la pantera sta al terzo, non segue più la sequenza classica. Il titolo è scritto in latino (Cetus hic pingitur et quomodo sustinet navem et gens et quomodo pisces intrant in os eius. Cetus diabolum significat et navis corpus hominis et nauta animam et arene maris divicias huius mundi et pisces animas); in francese il testo, ma con non poche intercalazioni latine. Interessante e originale risulta la scelta di spiegare le singole allegorie del racconto facendo a meno di riportarne tradizionalmente la trama:

La balena è il diavolo, / e il mare è questo mondo; / i granelli di sabbia sono / le ricchezze del mondo; / il marinaio è l'anima, / la nave che egli deve custodire è il corpo ; / e il fuoco è l'amore, / perché l'uomo ama come suo signore / il suo oro e il suo argento. / Quando il diavolo se ne accorge / e l'uomo si sente più sicuro, / allora lo annega. / E dice lo scritto: / la balena ha tale natura / che quando vuole mangiare / comincia a sbadigliare, / e sbadigliando / emette dalla sua bocca un profumo / tanto soave e tanto buono / che i piccoli pesci, / ai quali il profumo è gradito, / entrano nella sua bocca; / allora la chiude, / e li inghiotte. / E il diavolo ugualmente / inghiottirà la gente / che l'amerà tanto / da entrare nella sua bocca. / questo dice il Bestiario / un libro in latino. ${ }^{94}$

La versio B-Is è fonte anche del cosiddetto Bestiario (divino) redatto in versi, ancora in Inghilterra, tra il 1210 e il 1211 da Guillaume le Clerc (o le Normand), poeta normanno celebre per aver composto anche poesie morali e religiose; ${ }^{95}$ questa versione, molto elaborata, dedica 35 capitoli agli animali, rispettivamente i XXIV, XXV e XXVI per la pantera, la balena e la pernice.

Nous voulons vous parler maintenant d'un être extraordinaire qui vit dans la mer. Dans la mer, qui est grande et saine, vivent l'esturgeon, la balene, le turbot et le cachalot, et un grand poisson que l'on nomme marsouin. Mais il esiste aussi un mostre tout à fait étonnant, très malfaisant et très dangereux; il se nomme cetus en latin. Il est d'un voisinage néfaste pour les marins. Les écailles qui se trouvent au sommet de son dos ont tout à fait l'aspect

${ }^{94}$ Ed. e trad. Morini, 1996, pp. 212-215.

95 Cf. Orlandi, 1985, pp. 1096-1097; Bianciotto, 1980, pp. 67-69, e Dolcetti Corazza, 1992, pp. 71-72. 
de sable; quand il élève le dos au-dessus des flots, ceux qui naviguent dans les parages s'imaginent qu'il s'agit d'une île, mais leur espérance est trompée. A cause de la grandeur de la bête, les marins pourchassés par la tempête viennent y chercher refuge. Ils pensent avoir trouvé un endroit sûr; ils jettent l'ancre, lancent la passerelle, préparent le feu et font cuire leur repas; pour attacher solidement leur navire, ils enfoncent de grands pieux dans le sable qui, à leurs yeux, a tout à fait l'apparence de la terre. Puis ils font un grand feu, je vous l'assure; mais quand ce mostre sent la chaleur du feu qui s'embrase sur son dos, alors il plonge avec une grande rapidité au plus profond des eaux, faisant avec lui plonger le navire et périr tous les hommes. C'est dans le même piège que tombent les malheureux affligés dépourvus de foi qui ont mis toute leur confiance dans le Diable, et qui s'attardent et demeurent dans les oeuvres que le péché impose, et dont l'âme malheureuse s'afflige. Au moment où elle y prête le moins attention arrive le Perfide (puisse-t-il brûler du feu d'enfer!): lorsqu'il sent le pécheur solidement agrippé à lui, il le renverse et le plonge avec lui au plus profond de l'enfer: ceux qui vont en ce lieu ont perdu le salut éternel $[\ldots]{ }^{96}$

Agli anni immediatamente precedenti il 1218 risale la compilazione delle due versioni del Bestiario di Pierre de Beauvais (o le Picard), l'ultimo di area francese dedicato alla moralizzazione delle nature degli animali. La redazione più antica è la prima, più breve (38 capp.) e più fedele al modello latino, che molto probabilmente è quello della versio B-Is,${ }^{97}$ quanto alla seconda, la version longue (71 capp.), vi sono buone possibilità che si tratti di un rimaneggiamento operato tra il 1245 e il 1268 da autore ignoto; ${ }^{98}$ i capitoli 24,25 e 26 della versione breve sono dedicati ai tre animali secondo l'ordine canonico che vede la balena occupare il posto mediano, sebbene essa qui compaia con il nome di lacovie, quasi certamente una corruzione dello Jacoine del Viaggio di Benedeit: 99

Il existe une bête marine appelée lacovie; elle est très grande, et son cuir est recouvert d'un sable tout à fait semblable à celui que l'on trouve sur la rive de la mer. Cette bête fait émerger son dos au-dessus des flots de la mer, de telle sorte que ceux qui conduisent les navires croient qu'il s'agit d'une île

\footnotetext{
96 Trad. Bianciotto, 1980, pp. 105-107.

${ }^{97}$ Cf. Bianciotto, 1980, pp. 19-20; Dolcetti Corazza, 1992, pp. 73 ss.

${ }^{98}$ Cf. Morini, 1996, p. XVIII.

${ }^{99}$ Cf. McCulloch, 1956, pp. 100-101.
} 
recouverte de sable. Ils y abordent et mettent le pied sur la bête, ils enfoncent leurs pieux, et attachent leurs bateaux tout autour. Après cela, ils font un grand feu, et cuisent leurs aliments sur le sable tout comme ils le feraient sur la terre. Quand la bête sent et éprouve la chaleur du feu, elle plonge au plus profond de la mer et entraîne les navires avec elle. C'est ainsi qu'ils périssent. De la même manière sont conduits à la mort les mécréants et ceux qui ne sont pas capables de discerner la ruse du Diable, ainsi que ceux qui mettent leur espérance en lui, et qui s'attachent à ses oeuvres comme les marins ont lié leurs navires à la bête, tous ceux-là seront plongés dans le feu d'enfer éternel. ${ }^{100}$

In breve tempo, già nel corso del XIII secolo, la materia fisiologica comincia a essere sottoposta a riletture che mescolano sacro e profano, non di rado privilegiando gli aspetti mondani e cortigiani e facendo convergere simbolismo medievale, erotismo ed enciclopedismo. L'esempio più celebre è il Bestiario d'amore di Richard de Fournival (1201-ca. 1260), letterato e poeta di Amiens; a questo imborghesimento delle arti e dei costumi è radicalmente informato l'utilizzo dell'antica natura fisiologica della balena:

[...] non si sa di chi potersi fidare, e chi vuol stare in guardia dai malvagi stia in guardia da tutti. Vi è infatti chi garantisce di essere assolutamente leale ed è invece un perfido traditore. E io crederei di meno proprio a colui che mi desse più assicurazioni a parole. Giacché, dal momento che si dà tanta pena per essere creduto a parole, vuol dire che conosce qualche cosa tale da suscitare timore e che egli vuole usare a fini malvagi. Molti sono finiti male per aver avuto fiducia in gente che dava assicurazioni di questo genere. È quanto accade con una balena di una specie tanto grande che, quando tiene il dorso fuor d'acqua, i marinai che la scorgono pensano che sia un'isola perché ha la pelle straordinariamente somigliante alla sabbia marina; tanto che i marinai vi si accostano come se si trattasse di un'isola, vi si stabiliscono e vi fanno dimora per otto o quindici giorni, cuocendosi il cibo sul dorso della balena. Ma quando essa sente il fuoco, si tuffa trascinandoli negli abissi marini. Per questo io dico che ci si deve fidare meno di ciò che al mondo sembra più sicuro. Così accade alla maggior parte delle donne che si fanno un amico $[\ldots] .{ }^{101}$

100 Trad. Bianciotto, 1980, pp. 48-49.

${ }^{101}$ Fournival, 1987, pp. 87, 89. 
Notevole fortuna pure per il cosiddetto Physiologus Theobaldi (Bestiarius/Physiologus seu liber de naturis XII animalium), composto da soli dodici brevi exempla in versi. Conservatosi in un centinaio di manoscritti redatti tra il XII e il XIV secolo, del suo autore, difficilmente identificabile, sappiamo che fu abate nell'Italia settentrionale o centrale nel corso del secolo XI. Molto probabilmente, infatti, l'opera era utilizzata a scopi didattici in ambienti monastici, come si può evincere dalla sua brevità e dalla scelta di renderla in versi, evidentemente per facilitarne la memorizzazione. ${ }^{102}$ Le sue fonti principali sono soprattutto le due versioni latine $\mathrm{Y}$ e $\mathrm{B}$, ma è stato dimostrato che l'autore si è pure servito dell'opera di Isidoro, Gregorio Magno e dei celebri Dicta Chrysostomi, detti così perché attribuiti al santo antiocheno (Incipiunt dicta Johannis Chrysostomi de naturis bestiarum), ma in realtà redatti in Francia intorno al 1000 sulla base della versio B e pervenutoci grazie a codici tedeschi e austriaci non precedenti il XII secolo. ${ }^{103}$

Teobaldo ripropone l'assimilazione allegorica del cetus al grande pesce "infero" di Giona:

Est super omne pecus, quod vivit in equore, cetus, / Monstrum grande satis cum superextat aquis. / Prospiciens illum, montem putat esse marinum, / Aut quod in oceano insula sit medio. / Hic si quando famem, quam fert sepissime grandem, / Alleviare velit, callidus os aperit, / Unde velut florum se flatus reddit odorum, / Ad se pisciculos ut trahat exiguos: / Exiguos tantum, quoniam comprendere magnum / Perfectumque nequit, sed nec in ore premit. / Piscis pisciculos claudit, conglutit et illos: / Non sic, non sic jam sorbuit ille Jonam. / Si sit tempestas, cum vadit vel venit estas / Et pelagus fundum turbidat omne suum, / Continuo summas se tollit cetus ad undas: / Est promontorium cernere non modicum. / Huic religare citam pro tempestate carinam / Naute festinant, utque foras saliant. / Accendunt vigilem, quem navis portitat, ignem, / Ut se calefaciant aut comedenda coquant. / Ille focum sentit, tunc se fugiendo remergit, / Unde prius venit, sicque carina perit. / Viribus est Zabulus quasi cetus corpore

102 Vedi Orlandi, 1985, pp. 1101-1103, e Dolcetti Corazza, 1992, pp. 58-60.

${ }^{103}$ Fonte principale del Physiologus di area tedesca, fortunati come poche altre opere dello stesso genere, i Dicta presentano gli animali, mediamente 27, suddivisi in due gruppi (terra e acqua; aria), senza però, che è quel che qui interessa, riservare spazio alcuno alla balena; il testo edito da Friedrich Wilhelm (parte II, pp. 18-44) è stato poi sostanzialmente riprodotto da Maurer, 1967, pp. 75-95. Per questi dati, cf. Orlandi, 1985, pp. 1097-1100, e Dolcetti Corazza, 1992, pp. 56-59. 
magnus, / Ut monstrant magni, quos facit ille, magi. / Mentes cunctorum, qui sunt ubicunque, virorum / Esurit atque sitit, quosque potest, perimit. / Sed modicos fidei trahit in dulcedine verbi, / Namque fide firmos non trahit ille viros. / In quo confidit quisquis vel spem sibi ponit, / Ad Stiga cum rapitur, quam male decipitur. ${ }^{104}$

Questa versione sarà tradotta in Inghilterra nel Middle English Physiologus/Bestiary, contenuto nel ms. Arundel 292 della British Library (ca. 1250). ${ }^{105} \mathrm{Si}$ tratta di una versione dal carattere orale sostanzialmente fedele all'originale latino di Teobaldo, ma, come vedremo nel capitolo che ci interessa (VIII), non priva di originalità e valore artistico; ${ }^{106} \mathrm{da}$ segnalare, tuttavia, che nella sequenza narrativa la sezione relativa alla descrizione della natura dell'animale è seguita separatamente da quella sulla sua simbologia, indicata sotto il titolo di Significacio:

La balena è un pesce (Cethegrande is a fis), il più grande che ci sia in mare, tanto che anche tu, se la vedessi fluttuare, diresti che si tratti di un'isola che sta sulla sabbia del mare. Questo pesce, che è immenso, quando ha fame spalanca la bocca [segue descrizione della prima natura] Questo pesce abita negli abissi marini e vive sempre lì sano e salvo finché arriva il momento in cui la tempesta agita tutto il mare, quando l'estate e l'inverno sono in lotta. Non può dimorare lì, tanto è torbido il fondo del mare, non può stare lì in quel periodo, quindi risale su e sta a fior d'acqua immobile. Quando il tempo è cosi inclemente, le navi che sono sbattute in mare dalla tempesta (odiosa è per loro la morte, caro il vivere), si guardano intorno e vedono questo pesce. Pensano che sia un'isola, si rallegrano molto di ciò e con foga si dirigono in quella direzione. Attraccano le navi e salgono tutti su per accendere un bel fuoco, con la pietra focaia e l'acciarino, su questa meraviglia. Lei avverte il fuoco e, inabissandosi d'improvviso nel fondo, li fa annegare: li uccide tutti senza ferite.

Significato: Il diavolo è forte in volontà e potenza, come i maghi che possiedono le arti magiche. Egli suscita nell'uomo fame e sete e molte altre voglie peccaminose. Adesca gli uomini con il suo effluvio, chiunque lo segua troverà disgrazia. Questi sono i piccoli, deboli nella fede, i grandi non riesce ad attirarli, i grandi, intendo i saldi nella giusta fede nella carne

${ }^{104}$ Cap. VIII (ed. Eden, 1972).

105 Vedi l'introduzione di Dora Faraci alla sua edizione in traduzione italiana dell'intero documento (Faracci, 1990, pp. 7-36). Cf. anche Dolcetti Corazza, 1992, pp. 103-107.

106 Vedi Faraci, 1990, pp. 19-26. 
e nello spirito. Chiunque ascolti la dottrina del diavolo col tempo se ne pentirà amaramente; chiunque riponga in lui la speranza lo seguirà nello scuro inferno. ${ }^{107}$

L'inserzione del motivo della tempesta combinato con quello dell'isolabalena è presente anche nell'Historia orientalis dell'agostiniano Jacques de Vitry, vescovo di san Giovanni d'Acri e più tardi cardinale e vescovo di Frascati, morto nel 1240:

Cete autem omnium piscium maximum est: habet autem strictos oris meatus, unde non nisi parvos pisciculos deglutit, quos oris anhelitu evocans et ad se trahens in ventrem suum mittit. Cum autem tempestas oritur in mari attollit se fluctus. Quidam autem ceti ita magni sunt, quod insule vel montes videtur, quibus urgente tempestate naute naues religantes dum credunt se super solidam terram manere, ignes accendunt, quos bella sentiens, tam naues quam nautas tecum trait in profundum (cap. XC). ${ }^{108}$

Collegato alla versio B-Is, spesso contaminata con passaggi di Solino ${ }^{109}$ e Isidoro, ${ }^{110}$ è il secondo libro del De bestiis et aliis rebus, per convenzione chiamato $\mathrm{H}$, scritto nel XII secolo e erroneamente attribuito al teologo sassone Ugo di San Vittore (1096-1141). ${ }^{111}$ I capitoli si succedono secondo un ordine differente da quello del Physiologus; per quel che ci riguarda, tra i 36 exempla del libro in questione manca la pernice, mentre la pantera precede di gran lunga la balena (titolo: $D e$ aspidochelone, bellua aquatica, habente partem figurae aspidis, partem testudinis; cheloni chelone enim est testudo bellua marina; cujus etiam

${ }^{107}$ Faracci, 1990, pp. 74-79.

${ }^{108}$ Ed. Vitry, 1597, pp. 193-194. Il passaggio sarà ripreso quasi per intero da Tommaso di Cantimpré, come tra poco vedremo. Cf. Faraci, 1990, p. 151.

${ }^{109} \mathrm{Su}$ questo scrittore si tornerà direttamente.

${ }^{110}$ Cf. Carmody, 1938, pp. 153-159. Tuttavia, il relativo brano di Isidoro (Etymologiae, XII, 6, 7) è riprodotto fedelmente tra le notizie "scientifiche" che concernono la balena al terzo libro, il cui autore è sconosciuto e più tardo: Ballenae autem sunt immensae magnitudinis bestiae ab emettendo et fundendo aquas dictae. Caeteris enim bestiis maris magis vomit undas. Bó $\lambda \lambda \varepsilon v v$ enim Graece emettere et jaculari dicitur (III, 55, PL 177,105 C). Il passo isidoriano era già stato integralmente riprodotto alla metà del IX secolo da Rabano Mauro nello sterminato compendio enciclopedico De universo (VIII, 5, PL, 111, 237 C).

${ }^{111}$ Vedi McCulloch, 1956, p. 30, n. 32. Edizione in PL, 177, 55-164. 
in sequenti opere mentio fiet), che occupa l'ultimo posto, appena dopo il "diamante" e la "perla:

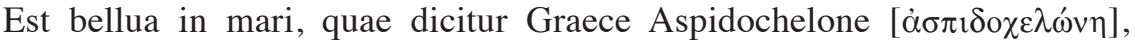
Latine autem aspidotestudo. Cetus autem est magnus, habens super corium suum tanquam sabuli seu arenae aggerem, juxta maris littus frequenter victitans. Haec in medio maris elevat dorsum suum super undas maris sursum, ita ut navigantibus nautis non aliud credatur esse quam insula, praecipue cum viderint totum illum locum sicut in omnibus littoribus maris sabulo esse obtectum. Putantes autem insulam esse, applicant navem suam juxta eam, et descendentes, figunt illic palos, et alligant navem, deinde ut coquant sibi cibos post laborem, faciunt ibi focos et ignes super arenam, quasi super terram. Illa vero, ut senserit ardorem ignis, subito mergit se in aquam, et navem secum trahit in profundum maris. Sic patiuntur omnes qui increduli sunt, et quicunque ignorant astutias diaboli, qui spem suam ponentes in eum, et operibus ejus se obligantes, simul merguntur cum illo in gehennam ignis ardentis. Ista est astutia ejus.

Secunda hujus belluae natura est haec. Quando esurit, aperit os suum, et quasi quemdam odorem suave olentem exhalat de ore suo, quem mox ut senserint minores pisces, congregant se intra os ipsius. Cum autem repletum fuerit os ejus diversis piscibus pusillis, subito claudit os suum, et transgluttit eos. Sic patiuntur omnes qui sunt modicae fidei. Voluptatibus enim ac lenociniis quasi quibusdam odoribus diabolicis inescati, subito absorbentur ab eo sicut pisciculi minuti. Majores enim se continent ab illo, neque appropriant ei. Sic etiam qui Christum semper in sua mente habent, magni sunt apud eum, et si sunt perfecti, agnoscunt multiformes astutias diaboli, et custodiunt se ab eo, et magis resistunt; ille vero fugit ab eis. Dubii autem et modicae fidei homines, dum vadunt post voluptates et luxurias diaboli, decipiuntur, dicente Scriptura: Unguento et variis odoribus delectantur (Prov., 27, 9). Et sic confringitur a ruinis anima. Cetus dicitur ab immanitate corporis. Habent enim ingentia corpora haec genera belluarum, aequalia montibus, in tantum ut etiam ibi naves quasi ad insulam applicentur, sicut ille qui excepit Jonam, cujus alvus tantae magnitudinis fuit, ut putaretur infernus, dicente ipso Jona propheta: De ventre inferni clamavi, et exaudisti vocem meam. ${ }^{12}$

Alla metà del XIII secolo l'utilizzazione del motivo secondo la versione del Physiologus latino interessò persino due noti enciclopedisti come il belga Thomas de Cantimpré (1201-1272) e il francescano Bartolomeo Anglico. Il primo lo riadattò nel Liber de natura rerum:

112 II, 36, PL, 177, 882 C-884 A. 
Cethe omnium piscium maximum est, ut Ysidorus dicit. Quem piscem educat mare quatuor iugerum magnitudinis. Habent strictos oris meatus, unde non nisi parvos deglutit pisciculos, quos odorifero oris anhelitu devorans et ad se trahens in ventrem suum mittit [...]. Itaque cum in mari tempestas exoritur, attollunt se super fluctus. ${ }^{113}$

Bartolomeo, perlopiù noto per il notevolissimo Liber de proprietatibus rerum, appellandosi all'autorità del misterioso Jorach (anche Jorath/ Iorat o Iuba/Giuba) $)^{114}$ - autore di un perduto trattato De animalibus ${ }^{115}$ citato da Plinio come fonte dell'esistenza di una balena lunga 600 piedi catturata in Arabia (Nat. Hist., XXXII, 10) - riadatta nel De genuinis rerum coelestium il racconto dell'isola-balena a nozioni scientifiche per le quali la balena parteciperebbe contemporaneamente delle nature animale, vegetale e minerale:

In hoc pisce [...] materia terrestris dominatur plus quam aqua, et ideo multe est corpulente et pinguedinis unde in senectute per magnitudine corporis in eius dorso coadunatur pulvis et condensatur in tantum quod herbe et frutices ibi crescuntur ita ut belua insule similis esse videtur; ad quam si navigantes incaute appropinquerint sine pericolo vix evadunt: nam aquam in tanta quantitate de ore eiecit, quod ipsam aliquando obruit et submergit (XII, 26). ${ }^{116}$

Nell' ambito del ramo didattico-enciclopedico è d'uopo ricordare anche il contributo in lingua d'oil del fiorentino Brunetto Latini (ca. 12201294), che assimila il motivo dell'isola-balena a quello della "balena" che inghiotte Giona:

Cetes est uns grans peissons que li plusor apelent balaine. Ce est uns peissons si granz comme une terre, qui maintes foiz remaint en sec, car il

${ }^{113}$ Liber de natura rerum,VI, 6 Cf. il brano poco sopra citato di Jacques de Vitry.

${ }^{114}$ Incerto autore di un trattato De animalibus citato anche da Vincent de Beauvais e Alberto Magno. Recentemente è stata proposta con discrezione l'identificazione con il re numida Giuba II, morto nel 23 d. C. (vedi Draelants, 2000, pp. 191-276). Alberto Magno, che lo riprende e lo usa come sua fonte per i brani sul grande pesce che blocca le navi, asserisce che sovente Jorach mente (De animalibus, XXII, 10b). Nondimeno, Jorath su quest'ultimo tema è pure la fonte di Plinio, Eliano, e, più tardi, di Alexander Neckam (1157-1217) e di Ruggero Bacone. Vedi Raven, 1968, p. 15.

115 Cf. Grant, 1999, p. 21.

${ }^{116}$ Citato in Faraci, 1991, p. 161. 
ne peut aller se non où la mer est haute. Ce est li peissons qui recut Jonam le prophete dedans son ventre, selonc ce que l'estoire dou Veil Testamente nos raconte, qu'il cuidoit estre alez en enfer por la grandor dou leu où il estoit [Jonas s'imaginait être arrivé en enfer, à cause de la grandeur du lieu où il se trouvait]. Cist peissons eslieve son dos en haute mer, et tant demore en un leu que li vent aporte sablon et ajostent sor lui, et i naist herbes et petiz arbrissiaus, por quoi li marinier sont deceu par maintes foiz là, cair il cuident que ce soit une isle, où il descendent, et fichent paliz et font feu; mais quant li peissons sent la chalor, il ne la puet sofrir, si s'en fuit dedanz la mer, et fait affondrer quanque il a sor lui (Trésor, I, 133). ${ }^{117}$

Una delle ultime manifestazioni del Physiologus latino è nel Libellus de natura animalium, conservato in due codici della Nazionale di Napoli risalenti al secolo XV:

Proprietas balene, que balena vocatur cetus, est ista quia stat et manet tantum in uno loco quod supra ipsam nascuntur ligna et herba, et sic naute, qui habent magnum desiderium quiescenti in terra, putant habere montem terre et lapidum et sic se pausant supra istam balenam et faciunt ignem supra ipsam, occasione satiandi cochinam et sic illa postquam calorem sentit demergit se in profundum et sic suffocantur omens naute desiderantes quiescere in illo loco.

Figura

Unde balena id est mundus iste; igitur omnes, credentes quiescere in mundo isto et requiem invenire, eorum fallacibus desideriis sunt decenti, quia mondana omnia sunt transitoria et caduca, iuxta illud Boetii: "Omne quod est genitum tendit ad interritum, id est ad mortem" (cap. XLI). ${ }^{118}$

Dal Libellus dipende strettamente il cosiddetto Bestiario valdese (titolo originario: Animanças o De les propietas de las animanças), trasmesso da due codici databili tra la fine del XV e gli inizi del XVI secolo conservati a Cambridge e Dublino. Così il testo del primo manoscritto in traduzione:

La proprietà della balena è tale, che essa permane tanto in un luogo, che sopra di lei nascono legna ed erbe, e così i marinai, che hanno grande desiderio di

${ }^{117}$ Ed. Chabaille, 1863, p. 186. La traduzione in francese moderno acclusa tra parentesi è citata in Bianciotto, 1980, p. 176.

118 A cura di Navone, 1983, p. 320; la citazione finale non è di Boezio (cf. MasperoGranata, 1999, p. 342). 
riposare in terra, pensano di aver trovato un monte di terra e di pietre, e così si riposano sopra di lei, e fanno fuoco; e poi che essa sente il calore del fuoco, si immerge nel profondo del mare, e tutti i marinai periscono. La balena significa questo mondo: dunque tutti quelli i quali credono di aver trovato riposo in questo mondo, sono ingannati nei loro falsi desideri, perché tutte le cose mondane sono passeggere e caduche. ${ }^{119}$

Nel 1984 è stata pubblicata la più aggiornata edizione curata da Anna Maria Raugei; insieme al testo originario, che segue, si dà anche quello del Libellus con poche varianti:

Proprietas balene est ista, quia manet tantum in uno loco quod supra ipsam nascuntur ligna et herbe; et sic naute qui habent magnum desiderium quiescenti in terra putant habere montem terre et lapidum; et sic se pausant super eam et faciunt ignem supra ipsam occasione faciendi cochynam; et sic illa, postquam ignis calorem sentit, demergit se cum illis in profundum maris; et sic suffocantur omnes naute desiderantes quiescere in illo loco. Unde balena id est mundus iste; igitur omnes credentes quiescere in mundo isto et requiem invenire eorum fallacibus desideriis sunt decenti, quia mondana sunt transitoria et caduca, iuxta illud Boetii: omne quod est genitum tendit ad interritum, id est ad mortem):

La propiota de la balena es aytal qu'ilh perman tant en un luoc que sobre ley nayson legnas e herbas; e enaysi li marinier que han grant desirier de repausar en terra pensan aver troba mont de terra e de peyras; e enaysi se repausan sobre ley e fan fuoc; e poys qu'ilh sent la calor del fuoc, se plomba al perfonç del mar, e tuit li marinier perisson. La balena significa aquest mont; dont tuit aquilh li cal creon aver troba repaus en aquest mont son engana en li lor fals desirier, car totas las cosas mondanas son trapassivols e cagivols. ${ }^{120}$

La tradizione fisiologica confluita nel Bestiario latino, sebbene mutuata dalle versioni francesi di quest'ultimo, ottenne grande attenzione anche in Italia, dove il successo di tale genere d'opere fu straordinario. ${ }^{121}$ Alla prima metà del XIII secolo risale il Bestiario toscano (o Libro della natura degli animali), pervenutoci in manoscritti del XIV secolo e derivato, insieme ad una redazione veneta, da un archetipo comune ora perduto:

\footnotetext{
119 Trad. Borghi Cedrini, 1977, p. 39.

${ }^{120}$ Raugei, 1984, pp. 315-316 e 223.

${ }^{121}$ Cf. Dolcetti Corazza, 1992, pp. 80-81.
} 
La balena si è uno pescie molto grande lo quale, quando s'asetta in alcuna parte e monstrase alcuna cosa sopra l'aqua, si credono li marinari, quando lo vedeno, che sia una insola; e or vi ligano li loro legni e montano suso a fare fuocho e altri loro facti. E quando lo pescie sente lo calore del fuocho, sí sse move e fa perire quelli homini e lo legno. Questo pescie significa questo mondo, che sí como la balena fa perire quelli che si fidano suso, lo somigliante fae questo mondo, che quando homo più vi se fida in questo misero mondo, tanto li viene piú tosto meno; ché noi vedemo che s'elli è imperatore u re u marchese u principi u conti u catani i qual vuole sia la degnitade temporale, sí vedemo che quantunque sono in magiore stato, che agevilemente nascie cosa là und'elli perden la signoria e la persona e l'avere e moglie e figliolo e tutti loro beni. E cosí divene a quelli del piccholo grado como a quelli del grande; e chiunque lo mundo l'escie di sotto si è mistieri ch'elli li faci perire. ${ }^{122}$

Da un unico testimone dei primi anni del XIV sec., proveniente da Gubbio e ora alla Nazionale di Roma (cod. 477), ci è noto il Bestiario moralizzato, sessantaquattro sonetti finalizzati all'applicazione delle nature degli animali alla morale:

Lo pescio ke se nomina balena / a la fiada sopra l'acqua pare [talvolta si rende visibile sopra il livello dell'acqua] / en semeliança d'isola terena, / lò o'va quelli k'è sopra mare [nel punto verso cui si dirigono i naviganti]; / pigliano posa è racollgniono alena, / conciano le cose da mangiare, / sentendo lo calore ella fera / tucta la gente fa pericolare. / Cotale semeliança à lo nemico / ke copre la sua malvasgitate / nello cospecto delli peccatori: / se a le fiade quale bene dicono / nolli sostene, tal n'à la niquitade, / somergeli e conduceli a li dolori (LIX). ${ }^{123}$

\section{Il Fisiologo anglosassone}

Fondato sulla contaminazione della versio B con $\mathrm{Y}^{124}$ il celebre Old English Physiologus è il primo a riferirci di una "balena verde" (vv. 1-49): 125

${ }^{122}$ Libro della natura degli animali, XXXIX, in Morini, 1996, p. 461.

${ }^{123}$ A cura di Carrega, 1983, pp. 153-154. Vedi pure Morini, 1996, pp. 522, 545, e Maspero-Granata, 1999, pp. 337-338.

${ }^{124}$ Dolcetti Corazza, 1992, pp. 99, 119-120.

${ }^{125}$ Cf. Vitry, 1914, p. 24: Dicitur autem quod in magno Oceano adeo sunt cete grandia, quod a nautis insule reputantur, maxime quando non mouentur et herbis atque aliis purgamentis maris operiuntur. Citato da Faraci, 1991, p. 160. 
E canterò ora del genere dei pesci, parlerò, come richiede l'arte del poetare, e secondo quanto la mia mente ricorda, della grande balena.

Pur senza volerlo, si imbattono in lei, terribile e crudele, gli uomini tutti

che percorrono i mari; è chiamata di nome Fastitocalon, lei che galleggia sulle antiche correnti.

D'aspetto è simile ad un masso scabro:

è come se, presso la riva del mare, fluttuasse, circondata da dune di sabbia, una massa enorme di alghe, tanto che i marinai credono d'aver posato gli occhi su una qualche isola; ed allora fissano le navi dall'alta prora a quella non-terra con le gomene dell'ancora, ormeggiano i destrieri del mare a riva, e poi sull'isola scendono baldanzosi; le imbarcazioni rimangono saldamente fissate sulla sponda, lambite dalla corrente.

Si accampano allora affaticati i naviganti, senza rendersi conto del pericolo, e sull'isola accendono il fuoco e alimentano una'alta fiamma; sono contenti gli uomini, anche se stanchi e desiderosi di riposi. Abile nell'ingannare, quando si accorge che i naviganti hanno saldamente preso dimora su di lei ed occupano quel luogo, felici del bel tempo, allora d'improvviso nelle onde salse con loro temerariamente s'immerge, l'ospite dell'oceano, raggiunge il fondo, e poi nell'antro della morte fa sprofondare e imprigiona nave e marinai. Allo stesso modo agiscono i demoni, allo stesso modo si comportano i diavoli. Senza posa, usando un oscuro potere, ingannano gli uomini e li incitano a desistere dalle buone azioni, li seducono a bella posta, tanto che questi cercano aiuto e conforto dai nemici, finché non scelgono di dimorare definitivamente presso il maligno.

Quando l'astuto perfido nemico si accorge 
dell'eterno tormento degli inferi, che i mortali

dell'umana specie nel suo recinto sono

saldamente tenuti, egli con perfidi inganni

si trasforma da quel momento in distruttore della loro vita, siano essi audaci o pavidi: sono quelli che peccando

hanno fatto qui la sua volontà, subito

protetto dall'elmo dell'invisibilità, si rifugia, ormai privato

[di ogni virtù,]

all'inferno, magma senza fine

sotto un'oscurità nebbiosa, proprio come la grande balena

che affonda gli uomini che percorrono i mari

e i destrieri delle onde. ${ }^{126}$

In questo frammento ${ }^{127}$ i marinai sbarcano sul dorso provenendo da est. Si tratta dell'uso più antico di tale racconto in area linguistica anglosassone.

Il Fisiologo antico-inglese (o anglosassone), opera in versi, è la più antica rielaborazione in una lingua germanica della versione latina e risale ad una età compresa tra la fine del secolo VIII e la seconda metà del X. All'XI e al XII secolo risalgono invece le due versioni alto-tedesche, seguite da quella islandese, degli inizi del XIII secolo. ${ }^{128}$

Nel testo antico-inglese, da un codice della Biblioteca della Cattedrale di Exeter -l'importantissimo Codex Exoniensis, redatto tra 960 e $980^{129}$ - leggiamo solo di tre animali, pantera (ff. 95v-96v), balena (96v$97 \mathrm{v}$ ), pernice (o pellicano ${ }^{130}$ ), con una tripartizione allegorica non casuale

${ }^{126}$ Una traduzione italiana è riproposta anche in Sanesi, 1998, pp. 63-65.

127 Il testo di questa redazione è infatti lacunoso, interrompendosi al verso $2 \mathrm{a}$ (f. 97v) della cosiddetta "pernice" per poi riprendere con le parole di Cristo al f. 98r (cf. D’Aronco, 1984, pp. 7-28p. 7, e Dolcetti Corazza, 1992, pp. 107 ss. e 143 per la traduzione).

${ }^{128}$ Cf. Dolcetti Corazza, 1992, p. 107; D’Aronco, 1984, p. 7-28, 7; Rossi-Reder, 1999, p. 462 .

${ }^{129}$ Codex Exoniensis (più comunemente noto come Exeter Book) 3501 (ff. 95v-98r), probabilmente donato alla cattedrale dal suo primo vescovo, Leofric, morto nel 1072, la cui compilazione ebbe luogo tra il 970 e il 990 ca. nell' area sassone occidentale dell'isola (cfr. Dolcetti Corazza, 1992, p. 107).

${ }^{130}$ Non è facile identificare l'uccello la cui natura è discussa nei sedici versi superstiti degli ottanta originari (vedi Dolcetti Corazza, 1992, pp. 107 ss.). 
finalizzata a richiamare i tre elementi terra, acqua, aria. ${ }^{131}$ Ciò fa del testo in questione un componimento unitario, ${ }^{132}$ nel quale la pantera diviene imago Christi, la balena di Satana (draca), il volatile degli uomini: ${ }^{133}$ la pantera come prototipo degli animali di terra (dēor), la balena per gli animali d'acqua (fisc), un uccello per gli animali dell'aria (fugol). ${ }^{134}$

A tal proposito è necessario che qui si menzioni una notizia riportata da Claudio Eliano a proposito della curiosa ostilità intercorrente proprio tra

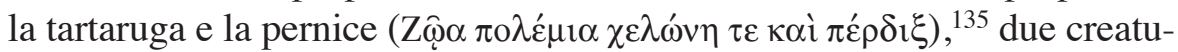
re che dunque possiederebbero nature contrarie anche in ambienti culturali estranei alla tradizione cristiana. Ciò testimonia, pertanto, dell'esistenza di un determinato linguaggio simbolico degli animali già notevolmente diffuso in ambienti mediterraneo-occidentali alla fine del II secolo; com'è noto, infatti, Eliano trascorse gran parte della sua vita a Roma.

Nella redazione greca del Physiologus la pernice occupa la sezione appena successiva all'aspidochelone, evidenziando una natura pure diabolica, attraverso la parafrasi di Ier., 17, 11: ${ }^{136}$

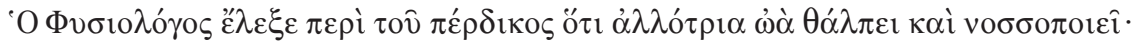

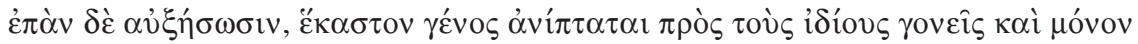

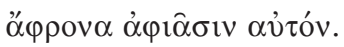

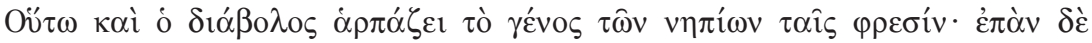

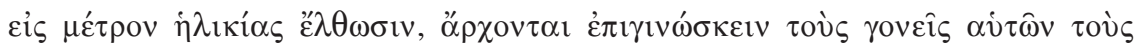

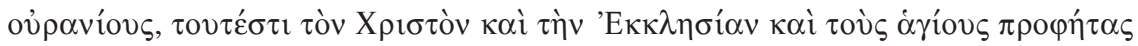

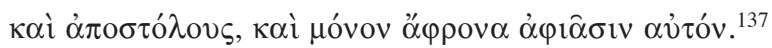

${ }^{131}$ Cf. D’Aronco, 1984, p. 8.

${ }^{132}$ Vedi Manganella, 1965, pp. 272-273.

133 Cf. D’Aronco, 1984, p. 8, n. 7, e Honegger, 1996, pp. 43 ss.; per una analisi linguistica e letteraria dei capitoli dedicati alla pantera e alla balena, si veda anche Diamond, 1970, pp. 192-204.

${ }^{134}$ Cf. Cometta, 2004, p. 13.

135 De natura animalium, IV 5, 1 (ed. R. Hercher, Leipzig, 1864, rist. Graz, 1971).

${ }^{136}$ Perdix fovit quae non peperit; fecit divitias, et non in iudicio; in dimidio dierum suorum derelinquet eas, et in novissimo suo erit insipiens.

${ }^{137}$ Physiologus Graecus, 18, ed. Sbordone, 1936. Così la traduzione italiana di Zambon, 2002, p. 57: "Il Fisiologo ha detto della pernice che cova le uova altrui e le fa schiudere: ma quando i piccoli sono diventati adulti, volano via, ciascuno secondo la sua specie, dai propri genitori, e la lasciano sola e confusa. Così anche il demonio rapisce la specie di coloro che sono piccoli nel senno: quando però sono pervenuti alla giusta mi- 
L'autore inserisce questa sezione subito dopo quella dell'aspidochelone con chiara consapevolezza, mirando a sottolineare la redenzione che Cristo e la Chiesa offrono a coloro che infine riescono a tenersi distanti dal demonio e dalle sue opere. Si tratta di una corrispondenza molto precisa e carica di significazioni. La sequenza "pantera - balena - pernice", come si vedrà, è riproposta nelle versioni B e Y latine (rispettivamente capp. 29, 30, 31; 23, 24 e 25), mentre nella $C$ è assente la pernice, sostituita dall'unicorno (pantera-balena: capp. 14 e 15).

L'autore scelse allora consapevolmente questo schema, anche perché i tre animali sono sempre introdotti con sequenze simili, con espressioni di ordine generale che riflettono tra l'altro lo stile poetico tipicamente antico-inglese: "ho udito / canto". ${ }^{138}$ Alla base v'è la specifica scelta dell' autore di creare un testo che non riproduca meccanicamente un modello, "ma in cui i tre exempla devono concorrere ad esprimere un'idea", ${ }^{139}$ a rappresentare in "triplice allegoria" i tre tipi Cristo, Satana e gli uomini. ${ }^{140} \mathrm{E}$ un poetare autonomo, in qualche modo individualistico, specchio della ferma consapevolezza dell'autore di comporre fuori dai parametri della sua fonte. Ciò lo si evince pure dall'assenza del riconoscimento dell'autorità del Physiologus -come suole definirsi l'autore della redazione greca- a vantaggio dell'inserimento di formule introduttive appunto prossime al lessico dell'epica germanica. ${ }^{141}$

In questa versione, la pantera vive in luoghi poco accessibili, "nelle cave spelonche dei monti", in una terra lontana e misteriosa che fa pensare al Regno dei Cieli (vv. 8 ss.). Di contro, la balena può spesso essere incontrata dai marinai, anche quando non la si cerchi. La prima caratteristica del cetaceo rilevata dal nostro testo è ovviamente la grandezza: "E canterò ora del genere dei pesci, parlerò, come richiede l'arte del poetare, e secondo quanto la mia mente ricorda, della grande balena [miclan ${ }^{142}$

sura dell'età, essi cominciano a riconoscere i loro genitori celesti, cioè Cristo e la Chiesa e i santi profeti e gli apostoli, e lo lasciano solo e confuso".

${ }^{138}$ Cf. D’Aronco, 1984, p. 17.

${ }^{139}$ Dolcetti Corazza, 1992, p. 114.

${ }^{140}$ Cf. Manganella, 1965, p. 272.

${ }^{141}$ Cf. Cometta, 2004, p. 15.

${ }^{142}$ Genitivo dell'aggettivo, comune e generico, micel, micla (cf. D'Aronco, 1984, p. 18; Dolcetti Corazza, 1992, p. 126). 
hwale $\left.{ }^{143}\right]$ ". ${ }^{144}$ Animale abbastanza noto alle genti dell'Europa settentrionale, esso, "tremendo e crudele" (v. 5), era dalle stesse molto temuto giacché considerato spietato come il demonio, il quale, sebbene indesiderato, costringe gli uomini ad incontrarlo. Il nome che qui gli si dà, Fastitocalon (vv. 6b-7a), è una chiara deformazione della forma Aspedocalon, da quella greca $\dot{\alpha} \sigma \pi \mathrm{i} \delta$ o $\chi \varepsilon \lambda \omega \dot{v \eta},{ }^{145}$ dunque direttamente da aspidochelone, riportata dal testo latino della versio B. ${ }^{146}$ Mostrandosi quasi come una sorta di pietra massiccia resistita alle antiche correnti del diluvio, ${ }^{147}$ la bestia presenta una "irregolarità morfologica" già rilevata in ambito esegetico da Eustazio di Antiochia con le seguenti

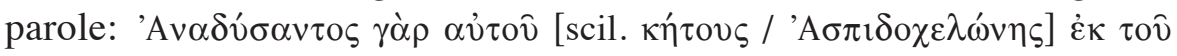

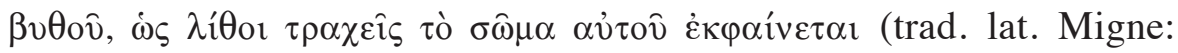
Ex profundo namque emergens eminet dorso multum supra aquas, quod duris asperisque saxis vestitum videtur). ${ }^{148}$ Simile immagine ci è fornita da san Girolamo, per quanto più concretamente connessa alla tartaruga con una combinazione delle due nature fisiologiche: Testudo marina, quae est grandis tamquam insula, dum emergit se de alto, ostendit se in ipso mari tamquam petram, nec se movet inde, quoadusque homines naves ligent. Et cum haec senserit, trahit se in altum. Sed cum

143 Antica denominazione germanica corrispondente ai moderni whale e walfish, al danese hval, al norvegese kval e all'islandese hvalur (cf. Dolcetti Corazza, 1992, p. 135, n. 332). Sull' etimologia di questi termini si tornerà più avanti.

144 Trad. di Dolcetti Corazza, in 1992, p. 135, vv. 1-3.

${ }^{145}$ Letteralmente "testuggine marina", lat. aspido testudo, ma utilizzato pure col significato generico di "grande mostro marino", dunque in seguito identificato con la balena (cf. G. Manganella, 1965, p. 276; Diamond, 1970, pp. 199 ss.; Dolcetti Corazza, 1992, p. 135). Nella forma fastaleon questo termine sarà ripreso nel XIII secolo da Tommaso di Cantimpré, enciclopedista che tratteremo più avanti (cf. Carus, 1880, pp. 173 e 200201). La leggenda del Fastitocalon fu riadattata da J. R. R. Tolkien, che la publicò con omonimo titolo nel 1962 come undicesimo dei sedici brevi poemi raccolti nel suo The Adventures of Tom Bombadill (pp. 112-115): "[...] Ah! foolish folk, who land on Him, / And little fires proceed to trim / And hope perhaps for tea! It may be that His shell is thick, / He seems to sleep; but He is quick, / And floats now in the sea / With guile; / And when He hears their tapping feet, / Or faintly feels the sudden heat, With smile / He dives, / And promptly turning upside-down / He tips them off, and deep they drown, / And lose their silly lives / To their surprise, / Be wise! [...]" (vv. 16-31).

${ }^{146}$ Ed. Carmody, 1941, p. 44.

${ }^{147}$ Cf. Cook, 1894, p. 65.

148 Commentarius in Hexaemeron, PG, 18, 723. 
esurierit, aperit os, et bonum odorem reddit: ad quem caeteri pisces colliguntur. ${ }^{149}$

Nel testo sassone, muovendosi la creatura alla superficie marina, ecco che intorno sembrano elevarsi colline sabbiose, ferme, fitte di alghe, sicché i marinai di passaggio la scambiano per un'isola e, senza sospetto alcuno, vi ormeggiano le proprie navi. Dopo aver acceso un fuoco, immediatamente vengono sballottati dal movimento e cadono così come le anime cadono vittime dei perversi e proditorii giochi del demonio. La balena, in sostanza descritta come masso scabro, fluttuante, circondata da dune di sabbia e a tratti ricoperta da masse di alghe, ${ }^{150}$ è creatura nella quale si incontrano, senza confondersi, il regno minerale (roccia) e quello vegetale (alghe). Risulta pertanto singolare il fatto che su questo pesce, così come pervenutoci nel testo anglosassone, la natura terrestre risulti dominare su quella acquea, un concetto bene espresso negli anni tra il 1220 e il 1230 nel cosiddetto Sermo Naturalium di Arnoldo il Sassone, il quale riportava a sua volta il pensiero non direttamente pervenutoci del solito, enigmatico Jorach: In hoc pisce magis terrestria quam aquea natura dominat. Nam quando senescit, colligit supra se radices fruticis et arbuste et herbarum, que crescunt super ipsum et multiplicantur. ${ }^{151}$ La presenza di vegetazione, qui fortemente voluta dall'anonimo autore, si manifesta ben radicata in un'antica tradizione che potrebbe originariamente essere rappresentata dal più antico testo del Physiologus, quello greco. ${ }^{152}$ Può allora trattarsi di un elemento molto popolare nelle Isole Britanniche già dal secolo VII, più che probabile periodo dell'arrivo del Fisiologo latino in Inghilterra, ${ }^{153}$ poi confluito nei bestiari del Basso Medioevo, nei quali, a proposito, si ha l'uso frequente di termini quali virgulta e arbusta.$^{154}$ Del resto, è stato appurato che $\mathrm{i}$ bestiari anglosassoni si rifanno perlopiù alla cosiddetta versio $\mathrm{B}$ del Fisiologo latino, oltreché all'opera di Ambrogio, Isidoro, Rabano Mauro; l'assenza del motivo della vegetazione in questi scrittori, come

${ }^{149}$ Epistola XVIII, ad Praesidium, 4, PL, 30, 187D.

${ }^{150}$ Sulle problematiche inerenti alla traduzione del termine saryrica, letteralmente da intendersi con "banco di giunchi"; vedi Faraci, 1991, pp. 156-157.

${ }^{151}$ Ed. Stange, 1905-1906, p. 63. Cf. Faraci, 1991, p. 157.

${ }^{152}$ Cf. Faraci, 1991, pp. 157 ss.

${ }^{153}$ Vedi Dolcetti Corazza, 1992, p. 101.

${ }^{154}$ Cf. Faraci, 1991, pp. 157-160. 
meglio vedremo, può spiegarsi con l'esistenza di quell'antico Physiologus greco di cui già si è sopra ipotizzato. ${ }^{155}$

Un primo accento va posto sulla meditata, esplicita assenza di erba, invece aggiungendosi della sabbia; tuttavia, risulta più interessante una discrepanza con quanto riferito nelle versioni greche e latine, vale a dire l'assenza dell'accensione del fuoco quale causa del movimento del pesce, il quale, mancando pertanto una minima consequenzialità, qui si mostra più consapevolmente perverso. T. $\mathrm{P}$. Campbell ha rilevato che al centro del racconto risiede certamente il tema della salvezza, fondato particolarmente sulla Lettera agli Efesini. ${ }^{156}$ L'autore, mediante l'immagine della balena, vuole avvertire gli uomini del pericolo rappresentato dal demonio e dai suoi inganni, finendo così per muoversi seguendo uno schema omiletico nel quale intenti lirici, tensione tropologica e chiavi allegoriche e/o anagogiche si fondono perfettamente. ${ }^{157}$ I marinai sono presentati come figurae instabilitatis, ${ }^{158}$ non super petram sed super harenam fundati sunt, ${ }^{159}$ per cui l'insieme tutto degli elementi naturali di volta in volta elencati, vale a dire l'erba, le alghe, gli arbusti, rivestono una esplicita funzione negativa, in quanto immagine di lussuria e concupiscenza, in una parola del saeculum, ${ }^{160}$ contrastando radicalmente con il ruolo "benevolo" degli alberi.

Così, quest'utilizzo specifico della vegetazione è da riconnettersi, nel suo significato originario, al celebre Mt., 11, 7 (Quid existis in desertum videre? Arundinem ${ }^{161}$ vento agitam?), che nell'esegesi ambrosiana dà la seguente sentenza: Harundines sumus, nulla ualidiores naturae radice fundati, et si leuis adspirauerit prosperioris aura successus, uago motum proximos uerberamus, inopes ad suffragandum, faciles ad nocendum. Harundines fluuios amant, et nos labentia mundi caduca

${ }^{155}$ Cf. Faraci, 1991, p. 160.

${ }^{156}$ Campbell, 1978, pp. 73-79, 78-79.

${ }^{157}$ Cf. Letson, 1979 , pp. 59-62

${ }^{158}$ Così li definisce Faraci, 1991, p. 161.

${ }^{159}$ Queste le parole di tre codici di altrettanti bestiari anglosassoni del secolo XIII (cf. Faraci, 1991, p. 162., n. 32).

160 Vedi gli esempi patristici (Gregorio Magno, Rabano Mauro) citati a modello da Faraci, 1991, pp. 164-165.

${ }^{161}$ Il summenzionato saryrica può infatti rendersi proprio con il latino arundinetum (Cook, 1973, p. 136; ma vedi pure Faraci, 1991, pp. 156-157 e 162 ss.). 
que delectant. ${ }^{162}$ E Gregorio Magno così ne inasprirà la valenza: Et quid per arundinem nisi carnalis animus designatur? ${ }^{263}$ Finché Rabano Mauro, riassumendone la sostanza, potrà concludere che: Arundo [...] significat mutabilitatem humanae mentis. ${ }^{164}$ Gli arbusti e le erbe sono elementi imperfetti, immagini degli uomini che aderiscono al male. Le insidie di Satana si manifestano attraverso gli inganni del cetaceo. Allora, la Lettera agli Efesini, come già anticipato, risulta basilare nell'elaborazione del testo tripartito, in particolare nel componimento finale, quello contenuto nel foglio 98r del codice di Exeter:

[...] bella / la parola che disse il principe della gloria: / "In qualunque momento voi, con la fede nel cuore, / ricorrerete a me, e desisterete / dai neri peccati degni dell'inferno, subito io / mi rivolgerò a voi con tenero affetto / ed animo benevolo. Voi sarete da allora in poi / riconosciuti e considerati da me come i gloriosi eletti, / come radiosi fratelli, reputati come figli". / Cerchiamo dunque di compiacere Dio con più fervore, / di avere in odio il peccato, di ottenere la protezione / e la benevolenza del Signore, finché il sole splende per noi, / in modo che, nella migliore tra le più eccellenti dimore, / nella bellezza della gloria, ci sia concesso di abitare. Finit. ${ }^{165}$

In questi versi della summenzionata epistola paolina è particolarmente forte l'eco della sezione 5, 10-14:

Probantes quid sit beneplacitum Deo: et nolite comunicare operibus infructuosis tenebrarum, magis autem redarguite. Quae enim in occulto fiunt $a b$ ipsis, turpe est et dicere. Omnia autem, quae arguuntur, a lumine manifestantur: omne enim, quod dicit: Surge qui dormis, et exsurge a mortuis, et illuminabit te Christus.

Il cristiano fugge le tentazioni del diavolo e raggiunge la meta ultima nell'abbraccio finale con Cristo.

Si palesa, allora, un contrasto netto con quanto è descritto nell'episodio di Jasconius, che è "isola" sassosa e priva di erba, dove la vegetazione è scarsa e manca la sabbia sulla riva, il contrasto si manifesta tra la pietra

\footnotetext{
162 Expositio Evangelii secundum Lucam, V, CChrSL, 14, 169.

${ }^{163}$ Homiliae in Evangelia, VI, PL, 76, 1096.

${ }^{164}$ De Universo, XIX, PL, 111, 521.

165 Trad. Dolcetti Corazza, 1992, p. 143.
} 
(insula petrosa) e la sabbia (nihil de arena fuit), e, soprattutto, tra gli alberi (silua rara) e l'erba (ulla herba); ma pure si stenta a scorgervi delle valenze ontologicamente negative.

Credo che non sia possibile stabilire un autentico rapporto di interdipendenza tra il testo irlandese e le varie redazioni del Fisiologo, ma è più facile ipotizzare che l'episodio della balena fosse così largamente diffuso nella cultura occidentale da originarsi in forme distintive secondo la natura del testo interessato. Certo, non si può negare un'influenza anche indiretta del primo sulla seconda, ma parimenti l'influenza si effettuò anche vicendevolmente, sino a giungere ai bestiari più tardi.

Ricordo infine, benché tardo e privo di originalità nella parte dedicata all'aspidochelone (è una riduzione della versio B latina), il noto Bestiary of Anne Walsh, redatto in Inghilterra intorno al 1420-1425 e conservato nella National Library of Denmark (ms. GKS $16334^{\circ}$ Kongelige Bibliotek): Est belua in mari que grece Aspidochelono dicitur quare navigantes insulam esse putantes applicant naves deinde faciunt sibi focos absorbentur a diabolo (f. 60). ${ }^{166}$

\section{Il Fisiologo islandese}

Fonte latina dell'ultima versione fisiologica qui analizzata è sostanzialmente, ancora, la versio $\mathrm{B},{ }^{167}$ di là da alcune divergenze che vedremo. In realtà le redazioni islandesi sono due (frammenti A e B), tuttavia coeve e simili per non pochi aspetti, sì che si suole parlare genericamente di Fisiologo islandese antico. ${ }^{168} \mathrm{Si}$ tratta dell'unica redazione fisiologica nella quale si riporti la presenza di un bosco sul dorso del cetaceo. Per quanto ne siano ormai appurati i legami con il testo inglese, ${ }^{169}$ è più che probabile che l'immagine del bosco derivi proprio dal capitolo decimo della Navigatio, ${ }^{170}$ opera da tempo già abbastanza popolare nell'Europa centro-settentrionale. E le notizie, e scientifiche e leggendari, sulle balene nei mari islandesi erano certo di pubblico

\footnotetext{
${ }^{166}$ Cf. Baxter, 1998, p. 147.

${ }^{167}$ Dolcetti Corazza, 1992, pp. 98 e 188 ss.; Zotto Tozzoli, 1992, pp. 15-16 e 43-44.

${ }^{168}$ Dolcetti Corazza, 1992, pp. 99, 188 ss.; Zotto Tozzoli, 1992, pp. 17 ss.

${ }^{169}$ Cf. Faraci, 1991, p. 169.

${ }^{170}$ Faraci, 1991, p. 169.
} 
dominio, come ci è attestato dalla sezione "Le meraviglie dell'Islanda" dello Speculum regale (antico norv. Konungs skuggsjá; cap. XII), ${ }^{171}$ una sorta di enciclopedia geografica e naturalistica compilata in Norvegia e pervenutaci in lingua islandese grazie ad un codice di Copenhagen redatto intorno al 1250 e infine tradotta in latino a partire dal Settecento. E in età medievale, dalle terre basche equipaggi colà si recavano per cacciarla, spingendosi sino alle coste della Groenlandia. ${ }^{172}$

La sezione dedicata al nostro animale è l'ottava del frammento B:

C'è nel mare una balena che si chiama aspedo ${ }^{173}$ e sul (suo) dorso è come se ci fosse un bosco. E in mezzo al mare solleva il suo dorso e i naviganti pensando che sia un'isola vi attraccano la loro nave e poi accendono il fuoco. Ma la balena avverte il calore e si inabissa in mare con tutti i naviganti.

Così vengono traditi gli uomini che ripongono la loro speranza nel diavolo, si rallegrano delle sue opere e affondano nei tormenti eterni con il demonio. ${ }^{174}$

La presenza di alberi potrebbe derivare da una traduzione esatta ma perduta dell'originale greco, anche se la versione islandese fu certamente redatta sul modello del testo latino, già presente nelle Isole Britanniche precedentemente al testo anglosassone, ${ }^{175}$ così come non può escludersi, ribadisco, una diretta influenza da parte della stessa Navigatio Brendani. ${ }^{176}$

\section{Conclusioni}

Sin dalla tarda antichità, il motivo fisiologico dell'aspidochelone destò l'interesse di esegeti e scrittori principalmente legati a contesti religiosi

${ }^{171}$ Ed. Geyser-Munch-Ungeruna, 1848, pp. 28-33 (una traduzione inglese di questo preziosissimo libro è in Larson, 1917).

${ }^{172}$ Cf. Delort, 1993, p. 34.

${ }^{173}$ Chiaramente variante abbreviata del latino aspidochelone già nella versio $B$ della redazione latina (ed. Carmody, 1939, p. 44).

174 Trad. di Del Zotto Tozzoli, 1992, p. 93.

${ }^{175}$ Cf. Dolcetti Corazza, 1992, pp. 99 ss. e 188 ss.

${ }^{176}$ Dolcetti Corazza, 1992, p. 199. 
cristiani che riuscirono nella non facile impresa di ereditare e custodire, valorizzare e riadattare quelle significazioni e quegli insegnamenti biblici propri e sostanziali al simbolismo animale in oggetto in quella che fu la sua epifania letteraria. La specificità e la natura di quel contesto alessandrino che generò la creatura del Physiologus, da quanto si è rilevato, indipendentemente dalla cultura dei singoli ambienti sociali che ne furono influenzati e dalle esigenze letterarie dettate da culture finanche discordanti da quella cristiana tardoantica, non manifestarono alcuna rilevante soluzione di continuità in un arco di tempo che investì e incorporò sia il mondo latino medievale, compresi i prodromi "laicoborghesi" dell'età umanistico-rinascimentale, sia il mondo europeo germanico in un periodo in cui quest'ultimo assisteva all'incontro e alla sintesi tra il paganesimo e la cristianità occidentale. Sulla base di quanto nel presente lavoro è stato esaminato e mostrato, è dunque possibile affermare che, attraverso la figura diaboli dell'aspidochelone, o, più grossolanamente, della balena o degli ipostatici mostri marini nei quali l'originaria creatura si è conservata, le realtà storico-religiose di volta in volta interessate hanno riconosciuto insegnamenti e disvelato immagini perfettamente corrispondenti all'archetipo alessandrino, ad un modello di cui le fonti testimoniano quanto fosse conosciuto e bene inteso, tra l'altro, anche nelle terre celtiche insulari, dove la tradizione manoscritta e l'acculturazione cristiana, principalmente delle età romanobarbarica e carolingia, avevano trasmesso approcci scritturistici di matrice esegetica continentale. L'intima dinamica dei testi esaminati, nella poligenesi delle immagini e delle forme, ha saputo conservare, nella secolare evoluzione socio-culturale, una "reductio anagogica" dell'ermeneutica tale da testimoniare che un testo sacro, pure sottoposto a processi narrativi "secolari", non si estingue, anzi sopravvive in forme che, combinando "sacro" e "profano", sono espresse ora nel mito, ora nell'epica, ora nel folklore, nel fantastico o nella lirica immaginativa del popolo. Un "simbolo delle origini", un archetipo, o un prototipo, che dir si voglia, nel processo temporale ha subito, e certo, un affievolirsi della coscienza sacrale che lo aveva generato, sopportando così di essere riadattato a speculazioni moraleggianti, lirismi cortigiani e miti popolari, e tuttavia, lo ribadisco, nulla ha potuto inficiarne l'origine sacrale, custodita dall'intaccato valore esegetico delle origini e dalla naturale e universale tensione allegorica dell'homo religiosus e dei Poeti d'ogni tempo. 


\section{BIBLIOGRAFIA}

Auber, C. A., Histoire et théorie du symbolisme religieux avant et depuis le christianisme, 4 voll., Paris, 1884.

BAXTER, R., Bestiaries and Their Users in the Middle Ages, Phoenix, 1998.

Bianciotto, G. (ed.), Bestiaires du Moyen Âge, Paris, 1980.

Borghi Cedrini, L., Appunti per la lettura di un bestiario medievale. Il Bestiario valdese. Schede linguistiche, Torino, 1977.

Brehaut, E., An Encyclopedist of the Dark Ages: Isidore of Seville, New York, 1912.

CAHIER, C. e A. Martin, Bestiaires, Textes, in Mélanges d'archéologie, d'histoire et de littérature, rédigés ou recueillis par les auteurs de la monographie de la cathédrale de Bourges (Charles Cahier et Arthur Martin), 4 tomes, 2 vols., Paris, 1847-1856: II (1851), III (1853), IV (1856).

CAmpbell, T. P., "Thematic Unity in the Old English Physiologus", Archiv für das Studium der Neueren Sprachen und Literaturen, 215, 1978, pp. 73-79, 78-79.

CANARD, M., "Le Conte de l'île-baleine et son utilisation dans la polémique byzantine contre les Pauliciens", Revue des Études Arméniennes, 9, 1972, pp. 379-384.

CANTIMPRATENsis, Th., Liber de natura rerum, Editio princeps secundum codices manuscriptos. Teil 1: Text, ed. H. Boese, Berlin-New York, 1973.

Carmody, F. J (ed. e versio), "Physiologus Latinus", University of California Publications in Classical Philology, 12, 1941, pp. 95-134.

_, "De bestiis et aliis rebus and the Latin Physiologus", Speculum, 13, 1938, pp. 153-159.

_, (ed.), Physiologus latinus: édition préliminaire. Versio B, Paris, 1939.

_, Quotations in the Latin Physiologus from Latin Bibles earlier than the Vulgata, Berkeley, 1944.

CARP, T., "The three late-coming Monks: Tradition and Invention in the Navigatio Sancti Brendani", Medievalia et Humanistica, 12, 1984, pp. 127-142.

CARrega, A. (cur.), Le proprietà degli animali, Genova, 1983.

CARUs, J. V., Histoire de la zoologie, depuis l'antiquité jusqu'au XIXe siècle, Paris, 1880.

Cattabiani, A., Volario. Simboli, miti e misteri degli esseri alati: uccelli, insetti e creature fantastiche, Milano, 2000; rist. 2001.

Cerulli, E., Atti di Giulio di Aqfahs, 2 voll., Louvain, 1959.

_, "La conquista persiana di Gerusalemme e altre fonti orientali cristiane di un episodio dell'Orlando furioso", Orientalia, 15, 1946, pp. 439-81.

_, Il Libro etiopico dei Miracoli di Maria e le sue fonti nelle letterature del Medio Evo latino, Roma, 1943.

Chabaille, P. (ed), Li livres dou Tresor, Paris, 1863.

Ciccarese, M. P. (cur.), Animali simbolici. Alle origini del Bestiario cristiano I (agnello-gufo), Bologna, 2002.

_. "Bibbia, bestie e Bestiari: l'interpretazione cristiana degli animali dalle origini al Medioevo", in G. Scianchi (cur.), Il Battistero di Parma. Iconografia, iconologia, fonti letterarie, Milano, 1999.

Cometta, M., La ricezione del Fisiologo nel mondo germanico, Milano, 2004. 
Conti, C., "Il Fisiologo etiopico", Rassegna di Studi Etiopici, 10, 1951, pp. 5-51.

Cook, A. S. (ed.), The Old English Physiologus, New Haven-London-Oxford, 1921 ; rist. 1973.

_, "The Old English 'Whale", Modern Language Notes, 9, 1894, pp. 65-68.

D’Aronco, M. A., "Considerazioni sul Physiologus antico inglese: Pantera vv. 8b13a; Balena vv. 1-7”, AION, Filologia Germanica, 27, 1984, pp. 7-28.

DELORT, R., "La balena: realtà e mito nel Medioevo", in Lo specchio oscuro: gli animali nell'immaginario degli uomini, Convegno internazionale: Genova, 16-17-18 novembre 1990, Museo Civico di storia naturale G. Doria, a cura di L. Battaglia, Torino, 1993, pp. 31-39.

Demus, O., "Bemerkungen zum Physiologus von Smyrna", Jahrbuch der österreichischen Byzantinistik, 25, 1976, pp. 232-257.

DiAmond, R. E., Old English Grammar and Reader, Detroit, 1970.

Doвschütz, E. von (ed.), Das Decretum gelasianum: de libris recipiendis et non recipiendis in kritischem Text herausgegeben und untersucht, Leipzig, 1912.

Dolcetti CoRAzZA, V., Il Fisiologo nella tradizione letteraria germanica, Alessandria, 1992.

Draelants, I., (ed.), Occident et Proche-Orient: contacts scientifiques au temps des croisades. Actes du Colloque de Louvain-la-Neuve, 24-25 mars 1997, Louvain-la-Neuve-Turnhout, 2000.

EdEn, P. T. (ed.), Theobaldi Physiologus, Köln-Leiden, 1972.

FARACI, D. (cur.), Il Bestiario Medio Inglese (Ms. Arundel 292 della British Library), L'Aquila-Roma, 1990.

_, "Considerazioni su parola e immagine nella tradizione dei bestiari medievali", in Faraci, 1991, pp. 9-36.

_, "Navigatio Sancti Brendani and its relationship with Physiologus", Romanobarbarica, 11, 1991, pp. 149-173.

_, Simbolismo animale e letteratura, Manziana (Roma), 2003.

FILONE, DI AlESSANDRIA, Tutti i trattati del commentario allegorico alla Bibbia, a cura di Roberto Radice, Milano, 1994.

Fournival, R. de, Il Bestiario d'amore, Parma, 1987.

Geyser, R., Munch, P. A. \& Unger, R. (ed.), Speculum Regale. Konungs-Skuggsjá. Konge-Speilet. Et philosophisk-didaktisk skrift fortattet i Norge mod slutningen af det tolfte aarhundrade, Christiania (Oslo), 1848.

GRANT, R. M., Early Christians and Animals, London, 1999.

Grässe, J. G. T., Die beiden ältesten lateinischen Fabelbücher des Mittelalters: des Bischofs Cyrillus Speculum Sapientiae und des Nicolaus Pergamenus Dialogus Creaturarum, Tübingen, 1880.

Grossi, V., "Il Decretum Gelasianum. Nota in margine all'autorità della Chiesa di Roma alla fine del sec. V", Augustinianum, 41, 2001, pp. 231-255.

Henkel, N., Studien zum Physiologus im Mittelalter, Tübingen, 1976.

Hommel, F., Die aethiopische Uebersetzung des Physiologus nach je einer Londoner, Pariser und Wiener Handschrift, herausgegeben, verdeutscht und mit einer historischen Einleitung versehen, Leipzig, 1877. 
_, "Die äthiopische Physiologus", Romanische Forschungen, 5, 1890, pp. 13-36. Honegger, T., From Phoenix to Chauntecleer. Medieval english animal poetry, Tübingen, 1996.

IVERSEn, E., The Myth of Egypt and its Hieroglyphs in European Tradition, Copenhagen, 1961.

James-RaOul, D., Dans l'eau, sous l'eau. Le Monde aquatique au Moyen Âge, Paris, 2002.

La Fenice e altri poemi anglosassoni, traduzione a cura di R. Sanesi, Milano, 1998. Land, J. P. N., Anecdota Syriaca IV, Leiden, 1875.

Lantschoot, A. van, "Fragments syriaques du Physiologus", Le Muséon, 72, 1959, pp. 37-51.

LARSOn, M. L, The King's Mirror, New York, 1917.

LAuchert, F., Geschichte des Physiologus, Strasbourg, 1889; rist. Genève, 1974.

Lemerle, P. e Redgate, The Armenians, Oxford, 1998.

Letson, D. R., "The Old English Physiologus and the homiletic tradition", Florilegium, 1, 1979, pp. 59-62.

Manganella, G., "Gli animali nella poesia anglossassone", AION, Filologia Germanica, 8, 1965, pp. 261-284.

Maspero, F.-A. Granata, Bestiario medievale, Casale Monferrato, 1999.

MAurer, F., Der altdeutsche Physiologus. Die Millstätter Reimfassung und die Wiener Prosa (nebst dem lateinischen Text und dem althochdeutschen Physiologus, Tübingen, 1967.

McCulloch, F., “Pierre de Beauvais' Lacovie”, Modern Language Notes, 71, 1956, pp. 100-101.

_, Medieval Latin and French Bestiaries, Chapel Hill, 1962.

Mirandola, L., Chimere divine. Storia del Fisiologo tra mondo latino e slavo, Bologna, 2001.

Morini, L., Bestiari Medievali, Parma, 1987; rist. Torino, 1996.

Muradyan, G., Physiologus. The Greek and Armenian Versions with a Study of Translation Technique, Leuven, 2005.

Navone, P. (cur.), Le proprietà degli animali, Genova, 1983.

Obolensky, D., The Bogomils: A Study in Balkan Neo-Manichaeism, Cambridge, 1948.

OfFermanns, D., Der Physiologus nach den Handschriften G und M, Meisenheim am Glam, 1966.

Orlandi, G., "La tradizione del Physiologus e i prodromi del Bestiario latino", in L'uomo di fronte al mondo animale nell'Alto Medioevo, Settimane di Studio del Centro Italiano di Studi sull'Alto Medioevo, XXXI, 7-13 aprile 1983, Spoleto, 1985, pp. 1057-1106.

PAPACHRYSSANTHOU, D., "Les Sources grecques pour l'histoire des Pauliciens d'Asie Mineure", Travaux et Mémoires, 4, 1970, pp. 1-67.

Pitman, J. H., "Milton and the Physiologus", Modern Language Notes, 40, 7, 1925, pp. 439-440.

Pitra, J. B. (ed. e trad.), Spicilegium Solesmense complectens Sanctorum Patrum anecdota hactenus opera, selecta e graecis orientalibusque et latinis codici- 
bus, 4 voll., Paris, 1852-1858 ; rist. Graz, 1965.

RAHNER, H., Miti greci nell'interpretazione cristiana (Griechische Mythen in christlicher Deutung, Zürich, 1957), Bologna, 1980; rist. 1990.

Raugei, A. M. (ed.), Bestiario valdese, Firenze, 1984.

Raven, C. E., English Naturalists from Neckam to Ray. A Study of the Making of the Modern World, New York, 1968.

Rossi-Reder, A., "Beasts and Baptism: A New Perspective on the Old English Physiologus", Neophilologus: An International Journal of Modern and Mediaeval Language and Literature, 83, 1999, pp. 461-477.

Runciman, S., The Medieval Manichee. A Study of the Christian Dualist Heresy, Cambridge, 1947; rist. 1982.

SANESI, R. (cur.), La Fenice e altri poemi anglosassoni, Milano, 1998.

Sbordone, F. (ed.), Physiologus graecus, Milano, 1936.

_, "La tradizione manoscritta del Physiologus latino", Athenaeum, 27, 1949, pp. 246-280.

_, I Bestiari e le rime amorose del secolo XIII, Napoli, 1944.

_, Ricerche sulle fonti e sulla composizione del Physiologus greco, Napoli, 1936.

Scotт, A., "The date of the Physiologus", Vigiliae Christianae, 52/4, 1998, pp. 430-441.

SElmer, C. (ed.), Navigatio sancti Brendani abbatis from Early Latin Manuscripts, Notre Dame (Indiana), 1959.

Sfameni Gasparro, G., "I dualismi medievali", in Storia delle Religioni, vol. 3 : Religioni dualiste e Islam, a cura di G. Filoramo, Bari, 1995, pp 69-98.

Stange, E. (ed)., Die Encyclopädie des Arnoldus Saxo, zum ersten Mal nach einem Erfurter Codex, Erfurt, 1905-1906.

Tolkien, J. R. R., Le Avventure di Tom Bombadill, trad. it. con testo inglese a fronte, Milano, 2000; rist. 2004.

Tychsen, O. G., Physiologus Syrus, Rostock, 1795.

Vitry, J. de, Die Exempla aus den Sermones feriales et communes, ed. J. Greven, Heidelberg, 1914.

_, Historia orientalis, ed. Fr. Moschus, Douay, 1597.

Voisenet, J., Bestiaire chrétien. L’imagerie animale des auteurs du Haut Moyen Âge (V-XI siècle), Toulouse, 1994.

Wellmann, M., Der Physiologus: eine religionsgeschichtlich-naturwissenschaftliche Untersuchung, Leipzig, 1930.

WentKer, S., Der arabische Physiologus, PhD dissertation, Universität Wien, 2004.

WILhelm, F., Denkmäler deutscher Prosa des XI und XII Jahrhunderts, München, 1914.

ZAmbon, F. (cur.), Il Fisiologo, Milano, 1975; rist. 2002.

_, L'alfabeto simbolico degli animali, Roma, 2001; rist. 2003.

Zотто Tozzoli, C. del, Il Physiologus in Islanda, Pisa, 1992. 